Pure and Applied Mathematics Quarterly

Volume 5, Number 2

(Special Issue: In honor of

Friedrich Hirzebruch, Part 1 of 2)

$571-618,2009$

\title{
Some Numerical Results in Complex Differential Geometry
}

\author{
S. K. Donaldson \\ Dedicated to Professor Friedrich Hirzebruch
}

\begin{abstract}
The first part of this paper discusses general procedures for finding numerical approximations to distinguished Kahler metrics, such as Calabi-Yau metrics, on complex projective manifolds. These procedures are closely related to ideas from Geometric Invariant Theory, and to the asymptotics of high powers of positive line bundles. In the core of the paper these ideas are illustrated by detailed numerical results for a particular K3 surface.
\end{abstract}

Key words: Calabi-Yau metric, Bergman kernel, numerical integration

\section{INTRODUCTION}

Far-reaching existence theorems, exemplified by Yau's solution of the Calabi conjecture for Kahler-Einstein metrics, are a distinctive feature of Kahler geometry. There are many open problems - the extension of Yau's results to the "positive" case and to Kahler Ricci solitons; existence theory for constant scalar curvature and extremal metrics; the study of the associated parabolic Ricci flow and Calabi flow equations - making up a very active and challenging research area today. Alongside this, little attention has been paid to the search for explicit numerical solutions: that is, good approximations to the metrics treated by the theory. The only step in this direction seems to be the pioneering and

Received December 12, 2005. 
recent work of Headrick and Wiseman [8]. In this article we discuss another approach to this question, illustrated by some numerical results for a particular K3 surface $S$; the double cover of the projective plane branched over the sextic curve $x^{6}+y^{6}+z^{6}=0$. The Kahler-Einstein metric $\omega$ on $S$ is characterised by the fact that, after suitable normalisation, the norm $|\theta|_{\omega}$ of the standard holomorphic 2 -form $\theta$ is equal to 1 at each point. We find a metric $\omega_{9}^{\prime}$ on $S$, given explicitly in terms of 26 real parameters, such that the norm $|\theta|_{\omega_{9}^{\prime}}$ differs from 1 by at most about $1.5 \%$ and on average, over $S$, by about .11\%. Thus there are good grounds for thinking that $\omega_{9}^{\prime}$ is a very reasonable approximation to the Kahler-Einstein metric, which could be applied to investigate any specific geometric question. The author has not yet attempted to investigate such applications in this case, except for some discussion of the spectrum of the Laplacian which we which give in Section 4. We will focus on this one example, although it should become clear that the methods could be applied, in a practical fashion, to other cases. The author has some other results for toric surfaces which he hopes to describe elsewhere.

In Section 1 below we describe a general approach to these approximation questions for projective algebraic manifolds. This approach is quite different from that of Headrick and Wiseman. The distinctive features are

- The use of metrics furnished by projective embeddings of the manifold.

- The construction of approximations to the differential-geometric solutions as limits of iterates of maps defined by integration over the manifold.

(It seems likely that these maps, which are dynamical systems on finite-dimensional spaces, can be viewed as discrete approximations to the Ricci and Calabi flows.)

To expand on the the first item above, consider an ample line bundle $L \rightarrow X$ over a compact complex manifold $X$, so for large enough integers $k$ the sections of $L^{k}$ give a projective embedding of $X$. Suppose $G$ is a positive definite Hermitian form on the vector space $H^{0}\left(L^{k}\right)$ of sections. Then there is a metric $h$ on the line bundle $L^{k}$ characterised by the fact that if $\left(s_{\alpha}\right)$ is any orthonormal basis of $H^{0}\left(L^{k}\right)$ then the function

$$
\sum_{\alpha}\left|s_{\alpha}\right|_{h}^{2}
$$

is constant on $X$. The curvature of the unitary connection on $L^{k}$ associated to $h$ has the form $-i k \omega_{G}$ where $\omega_{G}$ is a Kahler metric on $X$ in the class $2 \pi c_{1}(L)$. 
This metric can also be viewed as the restriction of the standard Fubini-Study metric on the projective space $\mathbf{P}\left(H^{0}\left(L^{k}\right)^{*}\right)$ (defined by the Hermitian form $G$ ) to the image of $X$ under the projective embedding. Thus we have a way to generate Kahler metrics from simple algebraic data-Hermitian forms on finite dimensional vector spaces.

The potential utility of this point of view has been advocated over many years by Yau, and a fundamental result of Tian [16] shows that this scheme does yield a way to approximate any metric. Tian proved that for any metric $\omega$ in the class $2 \pi c_{1}(L)$ there is a sequence $G_{k}$ of Hermitian forms such that

$$
\left\|\omega-\omega_{G_{k}}\right\|=O\left(k^{-2}\right),
$$

for any $C^{r}$ norm on $X$. Now the dimension of $H^{0}\left(L^{k}\right)$ grows like $k^{n}$ where $n=\operatorname{dim} X$, so - from a practical point of view - one might think that good approximations by these "algebraic" metrics would require the use of unduly large vector spaces (with a Hermitian metric depending on $O\left(k^{2 n}\right)$ real parameters). This point can be addressed by a refinement of Tian's result. There is a sequence $\tilde{G}_{k}$ such that

$$
\left\|\omega-\omega_{\tilde{G}_{k}}\right\|=O\left(k^{-\nu}\right)
$$

for any $\nu$. This is explained in the Appendix below. In other words, any metric can be very rapidly approximated by algebraic ones. (These approximations are in some ways analogous to the approximations of a smooth function defined by truncating the Fourier series.) Now suppose we are in a situation where we know (or hope) that a special metric $\omega$ (Kahler-Einstein, constant scalar curvature, extremal, Kahler-Ricci solution) exists. How can we generate a sequence of approximations to $\omega$ via Hermitian forms $\tilde{G}_{k}$, and can we get useful approximations with values of $k$ which are small enough to be manageable in practice? These are the questions we take up below.

The focus of this article is on the explicit numerical results. While there is already in place a considerable quantity of rigorous theory to back up these methods (and the authors interest in these questions grew out of work on abstract existence questions), there are also many points where more theory needs to be filled in; but we will not dwell on these here. It is also worth mentioning that many of these constructions are closely related to ideas in Geometric Quantisation Theory, and in particular the asymptotics of the classical limit, but we will not say more about this. 
Lacking any background in numerical methods, the author is not really qualified to comment on the comparison of the results here with those of Headrick and Wiseman. In one direction, the end-products seem comparable: approximations to Kahler-Einstein metrics on very special K3 surfaces with large symmetry groups using standard PC's. The author has not been able to directly compare the accuracy of the approximations in the two cases, but suspects that the best approximation we present here is comparable to the middle range of those achieved by Headrick and Wiseman and that by increasing $k$ a bit we would achieve something comparable to their best approximation. One fundamental disadvantage of our approach is that it is limited to algebraic varieties, whereas Headrick and Wiseman are able to vary the Kahler class continuously. One clear advantage, at least in the case studied here, is that we only need a few real parameters to store the metrics, as opposed to thousands or millions of parameters needed to record the values for a lattice approximation.

The author is grateful to Andrés Donaldson for instruction in programming. He would also like to mention the crucial part played by Appendix A ("Elementary programming in QBasic") of the text [1] in getting this project off the ground. Since its first appearance in preprint form, the article has been improved following comments from several people: two anonymous referees, Professors Liu and Ma (who elucidate some of the theory in their paper [10]) and Dr. Julien Keller (who also produced the figures in Section 2). The author thanks all of these mathematicians for the care they have taken. (We also mention here that the numerical results stated in this paper were obtained using programmes written in Basic. Keller has since rewritten the programmes in $\mathrm{C}++$, with an enormous gain in speed and accuracy. Thus the comments in the preprint version on the time required to perform the calculations have been deleted here, and the author hopes that this issue may be addressed by Keller elsewhere, along with other extensions of the ideas. All his programs relative to this work are available for download on the Institute for Mathematical Sciences website.) 


\section{General theory}

2.1. Constant scalar curvature. We return to the discussion of an ample line bundle $L \rightarrow X$, as in the previous section. Thus we consider the relations between two different kinds of metric data:

- Hermitian metrics $G$ on the finite-dimensional complex vector space $H^{0}\left(X ; L^{k}\right)$;

- Hermitian metrics $h$ on the line bundle $L^{k}$ such that the compatible unitary connection has curvature $-i k \omega_{h}$, where $\omega_{h}$ is a Kahler form on $X$. As a matter of notation, we write $G$ for the metric on $H^{0}\left(L^{k}\right)$ and $G^{-1}$ for the induced metric on the dual space. We also use the notation $G=\left(G_{\alpha \beta}\right), G^{-1}=G^{\alpha \beta}$ when working with a basis $\left(s_{\alpha}\right)$ of $H^{0}\left(L^{k}\right)$.

We have two fundamental constructions. Given a metric $h$ on $L^{k}$ we let $\operatorname{Hilb}(h)$ be the Hermitian metric on $H^{0}\left(L^{k}\right)$;

$$
\|s\|_{H i l b(h)}^{2}=R \int_{X}|s|_{h}^{2} d \mu_{h},
$$

where $d \mu_{h}$ is the standard volume form $\omega_{h}^{n} / n$ ! and $R$ is the ratio

$$
R=\frac{\operatorname{dim} H^{0}\left(L^{k}\right)}{\operatorname{Vol}\left(X, d \mu_{h}\right)}
$$

(which does not depend on the choice of $h$ ). In the other direction, given a Hermitian metric $G$ on $H^{0}\left(L^{k}\right)$ there is a metric $F S(G)$ on $L^{k}$ characterised by the fact that, for any orthonormal basis $s_{\alpha}$ of $H^{0}\left(L^{k}\right)$, we have

$$
\sum_{\alpha}\left|s_{\alpha}\right|_{F S(G)}^{2}=1
$$

pointwise on $X$. The Kahler form $\omega_{F S(G)}$ is the restriction of the standard FubiniStudy metric on projective space (defined by $G$ ) to $X$-regarded as a projective variety. Now we say that a pair $(G, h)$ is "balanced" if $G=\operatorname{Hilb}(h)$ and $h=$ $F S(G)$. (This terminology was introduced in [6]: the notion had been considered before by Zhang[17] and Luo [9].) Equally, since in this situation either of $h$ and $G$ determine the other, we can speak of a metric $h$ on $L^{k}$ or a metric $G$ on $H^{0}\left(L^{k}\right)$ being "balanced". Let $M$ denote the set of hermitian metrics on $H^{0}\left(L^{k}\right)$ and define $T: M \rightarrow M$ by

$$
T(G)=\operatorname{Hilb}(F S(G)) .
$$


Thus, by definition, a balanced metric is a fixed point of $T$. Now we have, from $[7]$

Proposition 1. Suppose that the automorphism group of the pair $(X, L)$ is discrete. If a balanced metric $G$ in $M$ exists then, for any point $G_{0} \in M$, the sequence $T^{r}\left(G_{0}\right)$ converges to $G$ as $r$ tends to infinity.

(In the original, preprint, version of [7] this convergence was raised as a question. In the version submitted for publication the convergence property was stated as a fact, but without a detailed proof. Meanwhile, independently, Y. Sano [15] supplied the detailed argument, in reponse to the original version of [7].)

We can spell out more explicitly the definition of the map $T$. Changing point of view slightly, let $z_{\alpha}$ be standard homogeneous co-ordinates on $\mathbf{C P}^{N}$ and $X \subset$ $\mathbf{C P}^{N}$ be a projective variety. Start with a positive definite Hermitian matrix $G_{\alpha \beta}$ and form the inverse $G^{\alpha \beta}$. For $z \in \mathbf{C}^{N+1}$ set

$$
D(z)=\sum G^{\alpha \beta} z_{\alpha} \bar{z}_{\beta} .
$$

Then the quotients

$$
f_{\gamma \delta}=\frac{z_{\gamma} \bar{z}_{\delta}}{D(z)},
$$

are homogeneous of degree 0 and can be regarded as functions on $\mathbf{C P}^{N}$, and so on $X$. Then the map $T$ is

$$
(T(G))_{\gamma \delta}=R \int_{X} f_{\gamma \delta} d \mu
$$

where $d \mu$ is the volume form induced by the Fubini-Study metric of $G$. Notice that the constant $R$ is chosen so that $G^{\gamma \delta} T(G)_{\gamma \delta}=N+1$. The geometry is unaffected by rescaling the metric $G$ so in practise we work with metrics normalised up to scale in some convenient but arbitrary way.

So far the parameter $k$ has been fixed; we now consider the effect of increasing $k$. The main result of [6] relates balanced metrics to constant scalar curvature metrics on $X$.

Proposition 2. Suppose that the automorphism group of the pair $(X, L)$ is discrete. If $X$ has a constant scalar curvature Kahler metric $\omega$ in the class $2 \pi c_{1}(L)$ then for large enough $k$ there is a unique balanced metric on $L^{k}$ inducing a Kahler 
metric $k \omega_{k}$ on $X$, and $\omega_{k} \rightarrow \omega$ as $k \rightarrow \infty$. Conversely, if there are balanced metrics on $L^{k}$ for all large $k$ and the sequence $\omega_{k}$ converges then the limit has constant scalar curvature.

Taken together, these two results give a procedure for finding numerical approximations to constant scalar curvature metrics. We choose a sufficiently large value of $k$ and then compute the iterate $T^{r}\left(G_{0}\right)$ for some convenient initial metric $G_{0}$ on $H^{0}\left(L^{k}\right)$. If $k$ is sufficiently large then the limit as $r$ tends to infinity is a good approximation to the differential geometric solution.

A crucial ingredient in the proof of Proposition 2 is the Tian-Yau-Zelditch-Lu expansion for the "density of states" function. If $s_{\alpha}$ is an orthonormal basis for $H^{0}\left(L^{k}\right)$ with respect to the standard $L^{2}$ metric we set $\rho_{k}=\sum\left|s_{\alpha}\right|^{2}$; a function on $X$ which does not depend on the choice of basis. Then if we take a fixed metric on $L$ and form the sequence of functions $\rho_{k}$ with the induced metrics on $L^{k}$ we have

$$
\rho_{k} \sim(2 \pi)^{-n}\left(k^{n}+a_{1} k^{n-1}+a_{2} k^{n-2}+\ldots\right),
$$

where the $a_{i}$ are local invariants and $a_{1}$ is $1 / 2 \pi$ times the scalar curvature.

2.1.1. A toy example. We take $X$ to be the Riemann sphere $\mathbf{C P}^{1}, L$ to be the line bundle $O(1)$. (Strictly this example does not fit into the framework above, since the pair has a continuous automorphism group $S L(2, \mathbf{C})$. However, the theory can undoubtedly be extended to relax the condition on the automorphisms, in the manner of [11], so we will ignore this technicality here.) A basis of $H^{0}\left(L^{k}\right)$ is given by $1, x, x^{2}, \ldots x^{k}$ where $x$ is a standard co-ordinate on $\mathbf{C}$. We restrict attention to $S^{1}$-invariant metrics, for the $S^{1}$ action $x \mapsto e^{i \theta} x$ on the sphere. The invariant metrics are represented by diagonal matrices in our basis, specified by the $k+1$ diagonal entries, thus

$$
D=\sum_{p=0}^{k} a_{p}|x|^{2 p}
$$

(Notice that the $a_{p}$ are really the entries of the metric $G^{-1}$ on the dual space: in practice it is easier to work with this rather than $G$.) The round metric on the sphere is given by $a_{p}=\left(\begin{array}{l}k \\ p\end{array}\right)$, when $D=\left(1+|x|^{2}\right)^{k}$. Due to the symmetry we know that for any $k$ the balanced metric is a standard round metric on the sphere. 
To make things even simpler, we can consider metrics invariant under the inversion $x \mapsto x^{-1}$, so $a_{p}=a_{k-p}$. Since the geometry is unaffected by a overall scaling $a_{p} \mapsto C a_{p}$ we have just $\lfloor k / 2\rfloor+1$ essential real parameters. In the first case, when $k=2$, one can evaluate the integrals in the definition of $T$ explicitly using elementary calculus. If we normalise so that $a_{0}=a_{2}=1 / 2$ and write $a_{1}=s$ the map is represented by the function

$$
\tau(s)=\frac{s \cosh ^{-1}(s)+\sqrt{s^{2}-1}\left(s^{2}-2\right)}{2 s \sqrt{s^{2}-1}-2 \cosh ^{-1}(s)} .
$$

The reader who plots this function will immediately see that the iterates $\tau^{r}\left(s_{0}\right)$ do indeed converge rapidly to the fixed point $s=1$. (The formula as written is valid in the range $s>1$ but has an obvious continuation to $s \leq 1$.)

We now consider the case $k=6$, and evaluate the integrals numerically. A typical sequence of iterates is indicated in the next table.

\begin{tabular}{|l||l|l|l|l|}
\hline $\mathrm{r}$ & $a_{0}$ & $a_{1}$ & $a_{2}$ & $a_{3}$ \\
\hline 0 & .018 & .495 & 4.5 & 54 \\
\hline 1 & .02833 & .8539 & 11.04 & 40.16 \\
\hline 2 & .03923 & 1.268 & 13.38 & 34.62 \\
\hline 3 & .05331 & 1.645 & 14.39 & 31.81 \\
\hline 4 & .07150 & 1.987 & 14.90 & 30.09 \\
\hline 10 & .2384 & 3.493 & 15.57 & 25.40 \\
\hline 20 & .7365 & 5.400 & 15.26 & 21.20 \\
\hline 30 & .9488 & 5.895 & 15.05 & 20.21 \\
\hline 40 & .991 & 5.983 & 15.01 & 20.03 \\
\hline$\infty$ & 1 & 6 & 15 & 20 \\
\hline
\end{tabular}

This confirms the convergence that the theory predicts, and similar resuts are obtained whatever initial values for $a_{0}, a_{1}, a_{2}, a_{3}$ are used. The choice of the particular initial values here is made because in this case all the metrics in the sequence can be represented as surfaces of revolution in $\mathbf{R}^{3}$, so one obtains a vivid representation of the evolution of the geometry of the surface through the sequence. The initial values give, roughly speaking, a connected sum of spheres joined by a small neck. The first application of $T$ stretches the surface into a long "sausage", which becomes convex after four more application of $T$, and thereafter the sausage slowly shrinks in length to approach a round sphere. See Figures 1-4. 


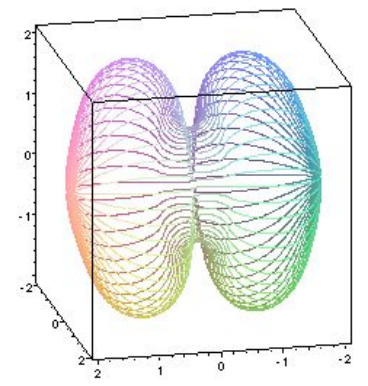

Figure 1. $r=1$

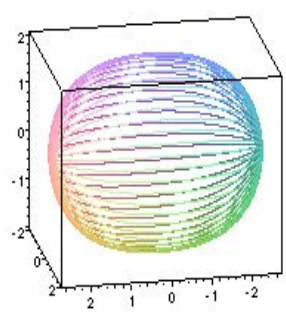

Figure 3. $r=10$

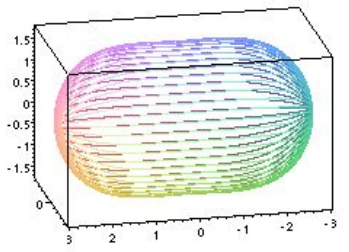

Figure 2. $r=5$

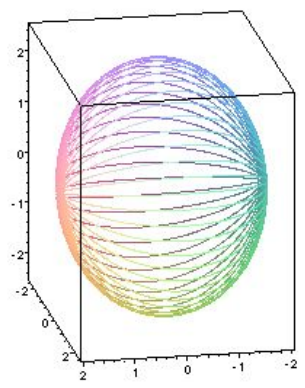

Figure 4. $r=40$

In this example we see that the convergence to the limit is, while steady, quite slow. Let $\epsilon_{i}(r)$ be the difference between $a_{i}(r)$ and the limiting value $a_{i}(\infty)$. From standard general theory we know that there is a constant $\sigma \in(0,1)$ associated to the problem such that for almost all initial conditions

$$
\epsilon_{i}(r) \sim c_{i} \sigma^{r}
$$

as $r \rightarrow \infty$. This constant $\sigma$ is just the largest eigenvalue of the derivative of $T$ at the fixed point, and the vector $c_{i}$ is an associated eigenvector. Analysing the sequence, one can see that in this case $\sigma$ is about .8. We also see numerically that the corresponding eigenvector has entries approximately

$$
(1,-2,-1,-4)
$$


The fact that one gets this eigenvector is easily explained by the $S O(3)$ invariance of the problem. The eigenvector corresponds to the second spherical harmonic on the sphere. This is given by

$$
f_{2}=\left(\frac{1-|x|^{2}}{1+|x|^{2}}\right)^{2} .
$$

Thus

$$
f_{2}=\frac{\left(1-|x|^{2}\right)^{2}\left(1+|x|^{2}\right)^{4}}{\left(1+|x|^{2}\right)^{6}}
$$

and

$$
\left(1-|x|^{2}\right)^{2}\left(1+|x|^{2}\right)^{4}=1-2|x|^{2}-|x|^{4}-4|x|^{6}-|x|^{8}-2|x|^{10}+|x|^{12} .
$$

A general point to make here is that while the convergence of the sequence $T^{r}$ is slow (since .8 is not much less than 1 ) it is easy, using this standard analysis of the linearisation, to define much more rapidly convergent sequences, and of course the same remarks hold in more complicated examples.

This toy model is the only example of the $T$-iteration which we will consider in this paper. The picture extends readily to the case of toric varieties in higher dimensions which, as mentioned before, the author hopes to take up elsewhere.

2.1.2. Extremal metrics. As we stated above, the results of [6] and [7] are limited, strictly, to the case where the pair $(X, L)$ does not have continuous automorphisms, but we anticipate that the theory can be extended to remove this condition. In this regard, we point out here that there is a straightforward modification which can be expected to produce numerical approximations to extremal metrics in the sense of Calabi [4]. Suppose that $(X, L)$ has a conected Lie group of automorphisms and fix a maximal compact subgroup $K$. Then $K$ acts on $H^{0}\left(X, L^{k}\right)$ and we restrict to $K$-invariant metrics. Then it may happen that the sequence $T^{r}\left(G_{0}\right)$ does not converge but that there is a sequence $g_{r}$ in the complexification of $K$ such that $g_{r}\left(T^{r}\left(G_{0}\right)\right)$ does converge, as $r \rightarrow \infty$. In this case, taking the limits, we again get a sequence of preferred metrics $\omega_{k}$ which we expect to converge to an extremal metric on $X$ as $k \rightarrow \infty$. While the theory here needs to be filled in, the procedure works effectively in the examples that the author has studied numerically. 
2.2. Calabi-Yau metrics. We now turn to the main topic of this paper: the case when the metric one wants to approximate is Ricci-flat. While this case could be treated within the constant scalar curvature theory outlined above, there is a slightly different, and simpler approach. Let $X, L$ be as before and suppose given a fixed smooth volume form $d \nu$ on $X$. A fundamental result of Yau asserts that there is a unique Kahler metric $\omega$ on $X$ in the class $2 \pi c_{1}(L)$ which realises a constant multiple of $d \nu$ as its volume form $\omega^{n} / n$ !. We describe a method for finding numerical approximations to this metric. The most interesting case is when $X$ is a Calabi-Yau manifold, so has a nowhere-vanishing holomorphic $n$-form $\theta$. Then the metric with volume form $d \nu=i^{n^{2}} \theta \wedge \bar{\theta}$ is Ricci-flat.

In a nutshell, we modify the definitions of the previous section by using the fixed volume form $d \nu$ in place of the Fubini-Study volume $d \mu$. Thus we say that a Hermitian metric $G$ on $H^{0}\left(X ; L^{k}\right)$ is $\nu$-balanced if $G=\operatorname{Hilb}_{\nu}(F S(G))$ where

$$
\|s\|_{\mathrm{Hilb}_{\nu}(\mathrm{h})}^{2}=R \int|s|_{h}^{2} d \nu .
$$

The existence of balanced metrics in this context is due to Bourguignon, $\mathrm{Li}$ and Yau [3], see also the recent paper [2]. It is closely related to well-known results relating moment maps to Geometric Invariant theory, and similar extensions were considered by Milson and Zombro [12]. In fact suppose that $\nu$ is any positive Radon measure on $\mathbf{C P}^{n}$. We say that a metric $G_{\alpha \beta}$ on $\mathbf{C}^{N+1}$ is $\nu$--balanced if

$$
G_{\gamma \delta}=R \int_{\mathbf{C P}^{N}} \frac{z_{\gamma} \bar{z}_{\delta}}{D(z)} d \nu
$$

When $\nu$ is derived from a smooth volume form supported on a projective subvariety $X \subset \mathbf{C P}^{N}$ this reproduces the previous definition. But we can also consider other measures, in particular, sums of point masses (or, in other words, the case when subvariety has dimension 0 ). Let us suppose that the measure $\nu$ satisfies one of the following two conditions:

(1) For any non-trivial linear function $\lambda$ on $\mathbf{C}^{N+1}$ the function $\log \left(\frac{|\lambda(z)|}{|z|}\right)$ on $\mathbf{C P}^{N}$ is $\nu$-integrable.

(2) $\nu$ is a sum of point masses supported on a finite set $Z$ and for any projective subspace $P \subset \mathbf{C P}^{N}$ we have

$$
\frac{\nu(Z \cap P)}{\operatorname{dim} P+1}<\frac{\nu(Z)}{N+1} .
$$


(The definition in the first item uses a metric on $\mathbf{C}^{N+1}$ but is clear that the condition does not depend on this choice.) It is easy to see that if $\nu$ is a smooth volume form on a subvariety $X$ which does not lie in any proper projective subspace then the first hypothesis holds.

Now we have

Proposition 3. If $\nu$ is a positive Radon measure on $\mathbf{C P}^{N}$ which satisfies either condition (1) or (2) above then there is a $\nu$-balanced metric on $\mathbf{C}^{N+1}$, and this is unique up to scale.

We recall the proof briefly. Note that the space of metrics $M$ is the symmetric space $G L(N+1, \mathbf{C}) / U(N)$ and there is a standard notion of geodesics in $M$; the images of analytic 1-parameter subgroups in $G L(N+1, \mathbf{C})$. For any non-zero vector $z \in \mathbf{C}^{N+1}$ we let $\psi_{z}$ be the function

$$
\psi_{z}(G)=\log |z|_{G^{-1}}^{2}+\frac{1}{N+1} \log \operatorname{det} G
$$

on $M$. The key point is that $\psi_{z}$ is convex on all geodesics. Changing $z$ by a scalar multiple only changes $\psi_{z}$ by the addition of a constant. Now given our Radon measure $\nu$ we set, with some abuse of notation,

$$
\Psi_{\nu}(G)=\int_{\mathbf{C P}^{N}} \psi_{z} d \nu(z)
$$

This is defined up to the addition of a constant; for example we can define $\psi_{z}$ for $z \in \mathbf{C} \mathbf{P}^{N}$ by taking the lift to a vector in $\mathbf{C}^{N+1}$ of length 1 with respect to some chosen reference metric. Now $\Psi_{\nu}$ is also convex, being a positive linear combination of convex functions. It is easy to check that a metric $G$ is $\nu$-balanced if and only if it is a minimum of $\Psi_{\nu}$. Such a minimum will exist so long as $\Psi_{\nu}$ is a proper function on $M$, and the convexity yields uniqueness. In turn, $\Psi_{\nu}$ is proper on $M$ if and only if it is proper on each geodesic. So what we have to verify is that - under either of the hypotheses (1), (2) - for each geodesic ray $G_{t}$ we have $\Psi_{\nu}\left(G_{t}\right) \rightarrow \infty$ as $t \rightarrow \infty$. Using the $G L(N+1, \mathbf{C})$-invariance of the problem it suffices to consider a geodesic of the form

$$
G_{t}=\operatorname{diag}\left(e^{\lambda_{\alpha} t}\right)
$$

where $\sum \lambda_{\alpha}=0$ and $\lambda_{0} \geq \cdots \geq \lambda_{N}$. Then

$$
\Psi_{\nu}\left(G_{t}\right)=\int_{\mathbf{C P}^{N}} \log \left(\sum e^{\lambda_{\alpha} t}\left|x_{\alpha}\right|^{2}\right) d \nu_{x}
$$


Consider first case (1). The first coefficient $\lambda_{0}$ must be positive and we have

$$
\log \left(\sum e^{\lambda_{\alpha} t}\right)\left|x_{\alpha}\right|^{2} \geq \log \left(e^{\lambda_{0} t}\left|x_{0}\right|^{2}\right)=\lambda_{0} t+\log \left|x_{0}\right|^{2} .
$$

By the hypothesis the term on the right hand side is $\nu$-integrable and so

$$
\Psi_{\nu}\left(G_{t}\right) \geq \lambda_{0} t \nu\left(\mathbf{C P}^{N}\right)+\text { Const. }
$$

and we see that $\Psi_{\nu}\left(G_{t}\right) \rightarrow \infty$ as required. In case (2) we write $\nu=\sum \nu_{i} \delta_{x^{(i)}}$, for points $x^{(i)}$ in $\mathbf{C P}^{N}$. Let

$$
\alpha(i)=\min \left\{\alpha \mid x_{\alpha}^{(i)} \neq 0\right\} .
$$

Then $\Psi_{\nu}\left(G_{t}\right) \sim c t$ as $t \rightarrow \infty$ where

$$
c=\sum \lambda_{\alpha(i)} \nu_{i}
$$

Elementary arguments, essentially the same as in usual Geometric Invariant Theory discussion in [14], [13], show that the condition that $c>0$, for all geodesics, is equivalent to the hypothesis (2).

Thus we know that, under the very mild hypotheses (1) or (2), $\nu$-balanced metrics exist. To find them, we make the obviuous modification to the algorithm of the previous section. We define $T_{\nu}: M \rightarrow M$ in just the same way as $T$ but using the measure $\nu$. That is, starting with a matrix $G$, we set

$$
T_{\nu}(G)_{\gamma \delta}=R \int_{\mathbf{C P}^{N}} \frac{z_{\gamma} \bar{z}_{\delta}}{D(z)} d \nu(z) .
$$

Notice that this is unaffected by rescaling $\nu$.

Proposition 4. Suppose $\nu$ satisfies either hypothesis (1) or (2). Then for any initial metric $G_{0}$ the sequence $T_{\nu}^{r}\left(G_{0}\right)$ converges to the $\nu$-balanced metric as $r \rightarrow$ $\infty$.

The proof is similar to that for the map $T$, but more elementary. We show that $T_{\nu}$ decreases the function $\Psi_{\nu}$; then the conclusion follows from the properness of $\Psi_{\nu}$. To make the notation simpler we will treat the case of point masses, the other case being essentially the same. To prove the inequality $\Psi_{\nu}\left(T_{\nu}(G)\right) \leq \Psi_{\nu}(G)$ we can without loss of generality suppose that $G$ is the metric given by the identity matrix. We can also suppose that the total mass $\sum \nu_{i}$ is 1 . We choose representatives $z^{(i)}$ in $\mathbf{C}^{N+1}$ with $\left|z^{(i)}\right|_{G^{-1}}=1$. Then

$$
\Psi_{\nu}(G)=\sum \nu_{i} \log \left|z^{(i)}\right|_{G^{-1}}^{2}+\frac{1}{N+1} \log (1)=0 .
$$


We treat the two terms in the definition of $\Psi_{\nu}$ :

$$
\Psi_{\nu}(T(G))=\sum \nu_{i} \log \left|z^{(i)}\right|_{T_{\nu}(G)^{-1}}^{2}+\frac{1}{N+1} \log \operatorname{det} T_{\nu}(G),
$$

separately. By the concavity of the logarithm function

$$
\sum_{i} \nu_{i} \log \left|z^{(i)}\right|_{T_{\nu}(G)^{-1}}^{2} \leq \log \left(\sum \nu_{i}\left|z^{(i)}\right|_{T_{\nu}(G)^{-1}}^{2}\right) .
$$

Now

$$
\sum_{i} \nu_{i}\left|z^{(i)}\right|_{T_{\nu}(G)^{-1}}^{2}=\sum_{i, \alpha, \beta} \nu_{i} z_{\alpha}^{(i)} \bar{z}_{\beta}^{(i)}\left(T_{\nu}(G)\right)^{\alpha \beta}
$$

but this is

$$
\sum_{\alpha \beta} \frac{1}{N+1} T_{\nu}(G)_{\alpha \beta} T_{\nu}(G)^{\alpha \beta}
$$

which is 1 , since $T_{\nu}(G)_{\alpha \beta}, T_{\nu}(G)^{\alpha \beta}$ are inverse matrices. So the first term in the definition of $\Psi_{\nu}$ is less than or equal to 0 . For the second term, the arithmeticgeometric mean inequality for the eigenvalues gives

$$
\frac{1}{N+1} \log \operatorname{det} T_{\nu}(G) \leq \log \left(\frac{\operatorname{Tr}\left(T_{\nu}(G)\right)}{N+1}\right)
$$

and the term on the right is zero, since

$$
\operatorname{Tr}\left(T_{\nu}(G)\right)=\sum_{i} \nu_{i}\left|z_{i}\right|_{G^{-1}}^{2}=\sum \nu_{i}=1
$$

Putting the two terms together we have $\Psi_{\nu}\left(T_{\nu}(G)\right) \leq 0$, as required.

To sum up, given a volume form $\nu$ on our algebraic variety $X$ we have, for each $k$, an algorithm for finding the $\nu$-balanced metric on $H^{0}\left(X ; L^{k}\right)$. Moreover, this is robust in the sense that if we approximate $\nu$ by another measure $\nu^{*}$ which is a sum of point masses - as we have to do in numerical integration - the numerical algorithm defined by $T_{\nu^{*}}$ will converge to a $\nu^{*}$-balanced metric provided only that $\nu^{*}$ satisfies the very mild condition (2) (which will happen for any reasonable approximation). Taking the restriction of the Fubini-Study metric and scaling by $k^{-1}$ we get a $\nu$-balanced Kahler metric $\omega_{k, \nu}$ on $X$. Now let $k$ tend to infinity. The same argument as used in the proof of Proposition 2, appealing to a variant of the asymptotic expansion result for the density of states function furnished by Dai, Liu and Ma [5], shows that the Kahler metrics $k^{-1} \omega_{k, \nu}$ converge to the metric with volume form $\nu$. Thus we have another procedure for finding numerical 
approximations to Calabi-Yau metrics. Experimentally at least, this converges more quickly than the more general "T-algorithm", which is probably related to the fact that the constant scalar curvature equation is of fourth order in the Kahler potential while the Calabi-Yau equation is a second order Monge-Ampere equation.

To give a toy example, consider again the $S^{1}$ invariant metrics on $\mathbf{C P}^{1}$ and the sections of $O(6)$. Let $\nu$ be the volume form of the standard round metric. Then, with the same starting point as before, we obtain the iterates of $T_{\nu}$ shown in the following table.

\begin{tabular}{|l||l|l|l|l|}
\hline$r$ & $a_{0}$ & $a_{1}$ & $a_{2}$ & $a_{3}$ \\
\hline 0 & .018 & .5 & 4.5 & 54 \\
\hline 1 & .1395 & 2.599 & 15.20 & 28.12 \\
\hline 2 & .4200 & 4.420 & 15.54 & 23.23 \\
\hline 3 & .6920 & 5.297 & 15.30 & 21.41 \\
\hline 4 & .8568 & 5.697 & 15.14 & 20.61 \\
\hline 10 & .9992 & 5.998 & 15.00 & 20.00 \\
\hline 13 & .9999 & 6.000 & 15.00 & 20.00 \\
\hline
\end{tabular}

The convergence is much faster. The parameter $\sigma$ governing the asymptotic behaviour is now about 42 (with the same eigenvector).

2.2.1. Refined approximations. In either variant of the theory, the balanced metrics or $\nu$-balanced metrics cannot usually be expected to give very close approximations to the desired differential-geometric solutions for practical values of $k$. An analysis of the convergence, using the Zelditch expansion, would probably show that the convergence is only $O\left(k^{-1}\right)$ or $O\left(k^{-2}\right)$. We now return to the issue raised in the Introduction of finding rapidly convergent approximations. We restrict the discussion here to the Calabi-Yau case. Thus we suppose that we have a metric $G_{0}$ on $H^{0}\left(X ; L^{k}\right)$ inducing a Fubini-Study metric $\omega_{G_{0}}$ on $X$, and that the volume form $d \mu_{G_{0}}=\frac{1}{n !} \omega_{G_{0}}^{n}$ is reasonably close, but not extremely close to the given volume form $\nu$ (which we assume to be normalised so that the total volumes are equal). We set $\eta=d \mu_{G} / \nu$; a function on $X$ which is close to 1 . We would like to "refine" $G_{0}$ to get a better approximation. This is essentially a linear problem. In the standard differential geometric theory we would consider a nearby Kahler metric of the form $\omega_{G_{0}}+i \bar{\partial} \partial \phi$. The linearisation of the volume 
form map is one half the Laplacian of $\left(X, \omega_{G_{0}}\right)$ so we would take

$$
\phi=-2 \Delta^{-1}(\eta-1)
$$

where $\Delta^{-1}$ is the Green's operator. Then, provided that $\omega_{G_{0}}$ is a good enough approxiation for the linearisation to be valid, the metric $\omega_{G_{0}}+i \bar{\partial} \partial \phi$ would be a better approximation to the desired Calabi-Yau metric and, iterating the procedure, we would generate a sequence which converged to that limit. Thus the question we address here is how to implement a procedure like this numerically, staying within the class of the "algebraic" Kahler metrics.

Let $G$ be any metric on $H^{0}\left(L^{k}\right)$ and $s_{\alpha}$ be a basis of sections. Then we have functions $f_{\alpha \beta}=\left(s_{\alpha}, s_{\beta}\right)$ on $X$, where $($,$) denotes the pointwise inner product$ on $L^{k}$ induced by $G$. Define " $\eta$ - coefficients"

$$
\eta_{\alpha \beta}=R \int_{X} f_{\alpha \beta}(\eta-1) d \nu
$$

(Notice here the diagonal terms $\eta_{\alpha \alpha}$ have a particularly simple interpretation, when $G$ is the balanced metric and the basis is orthonormal. In that case the integral of the positive function $f_{\alpha \alpha}$ is $1 / R$ and the numbers $\eta_{\alpha \alpha}$ can be viewed as a collection of mean values of $\eta-1$, weighted by $R f_{\alpha \alpha}$.) A natural criterion to define an "optimal" algebraic approximation to the differential-geometric solution is that all the coefficients $\eta_{\alpha \beta}$ vanish. Thus we say that a metric $G$ near to the balanced metric $G_{0}$ is a "refined approximation" if this occurs. One could hope to prove that, for large enough $k$, these refined approximations exist and (with a suitable interpretation of "close") are unique. Further, it is reasonable to expect that the resulting sequence of refined approximations would be very rapidly convergent, in the manner discussed in the Introduction.

In more invariant terms, we define a vector space $H\left(L^{k}\right)$ to be the Hermitian forms on $H^{0}\left(L^{k}\right)$. Then, given $G_{0}$, we have maps

$$
\iota: H\left(L^{k}\right) \rightarrow C^{\infty}(X) \quad \pi: C^{\infty}(X) \rightarrow H\left(L^{k}\right)
$$

defined in terms of a basis by

$$
\iota\left(\left(a_{\alpha \beta}\right)\right)=\sum a_{\alpha \beta} f_{\alpha \beta},
$$

and

$$
(\pi(F))_{\alpha \beta}=R \int_{X} F f_{\alpha, \beta} d \nu
$$


Then the condition we are considering is that $\pi(\eta-1) \in H\left(L^{k}\right)$ should vanish, which is the same as saying that $\eta-1$ is orthogonal in the $L^{2}$ sense to the finite dimensional subspace $\operatorname{Im}(\iota) \subset C^{\infty}(X)$.

The practical question we now face is: how can we find the refined approximations numerically, starting from the $\nu$-balanced metrics? As we have explained above this is basically a linear problem. If we set $G=G_{0}+h$ then we have a map

$$
V: h \mapsto\left(\eta_{\alpha \beta}\left(G_{0}+h\right)\right)
$$

from hermitian matrices $h$ to hermitian matrices $\eta_{\alpha \beta}$. We want to find a zero of $V$ and the standard procedure would be to invert the linearisation. This linearisation is given by a 4 -index tensor $S_{\alpha \beta \gamma \delta}$ with

$$
S_{\alpha \beta \gamma \delta}=\int_{X} f_{\alpha \beta}\left(f_{\gamma \delta}+\frac{1}{2} \Delta f_{\gamma \delta}\right) d \nu .
$$

If we compute $S$ and invert the corresponding matrix we could define an iterative procedure which ought to converge to the refined approximation. An obstacle to carrying this through is that (even when reduced by symmetry) the tensor $S$ has very many components so is relatively hard to compute in practice. Thus the author has not yet tried to implement this scheme but has used the following simpler procedure instead. Starting with an approximation $G_{0}$ we compute the error matrix $E=\left(\eta_{\alpha \beta}\right)$ and simply set

$$
G_{1}=G_{0}-\kappa E
$$

where $\kappa$ is a suitable positive constant. In fact we compute with the inverse metrics $G_{0}^{-1}$ and, since $E$ will be small, use the approximation

$$
G_{1}^{-1}=G_{0}^{-1}+\kappa G_{0}^{-1} E G_{0}^{-1} .
$$

Iterating this procedure yields a sequence which appears to converges reasonably well, although slowly, to the refined approximation, see the examples and discussion in the next Section. In Section 4 we give some further discussion and theoretical justification for this procedure.

Of course the whole theory sketched here needs to be developed properly, and better methods found. But we hope it will yield systematic procedures for finding improved numerical approximations, starting with the balanced metrics. 
2.2.2. Non-zero cosmological constant. We now consider the problem of finding approximations to Kahler-Einstein metrics with non-zero scalar curvature. Of course this can be fitted into the constant scalar curvature theory described above, but there is also a natural variant of the Calabi-Yau construction above which probably yields a simpler approach (although the author has neither attempted to develop the theory of this nor studied substantial examples numerically).

We suppose that our positive line bundle $L$ is either the canonical bundle $K$ of $X$ or its dual $K^{-1}$. Write $p= \pm k$ in the two cases, so our space of sections is $H^{0}\left(X ; K^{p}\right)$. Given a metric on $H^{0}\left(X ; K^{p}\right)$, let $s_{\alpha}$ be an orthornormal basis and set

$$
\phi=\sum s_{\alpha} \otimes \bar{s}_{\beta}
$$

This is a section of the bundle $K^{p} \otimes \bar{K}^{p}$ over $X$ which does not depend on the choice of orthonormal basis. Moreover if the sections of $K^{p}$ generate the fibers, which we can suppose is the case, $\phi$ does not vanish on $X$. Then $\phi^{1 / p}$ is a welldefined volume form on $X$. Using this volume form in place of the Fubini-Study form we get the notion of a canonically balanced metric on $H^{0}\left(X ; K^{p}\right)$. Likewise, using this volume form we define another variant $T_{K}$ of the map $T$. Of course we hope that if a Kahler-Einstein metric exists then it is the limit of canonically balanced metrics, and that the iterates of $T_{K}$ converge to the canonically balanced metrics. Furthermore, we can combine this discussion with that in (2.1.2), in the case when $X$ has continuous automorphisms, and we can hope to find KahlerRicci solitons in appropriate cases. But we leave all of this as a programme for the future, except to give here another toy example. If we take invariant metrics on $\mathbf{C P}^{1}$ and sections of $O(6)=K^{-3}$ we get the sequence of iterates of $T_{K}$ :

\begin{tabular}{|l||l|l|l|l|}
\hline$r$ & $a_{0}$ & $a_{1}$ & $a_{2}$ & $a_{3}$ \\
\hline 0 & .018 & .5 & 4.5 & 54 \\
\hline 1 & .0681 & 1.714 & 14.20 & 32.04 \\
\hline 2 & .1860 & 3.120 & 15.50 & 26.39 \\
\hline 3 & .3673 & 4.178 & 15.57 & 23.78 \\
\hline 4 & .5598 & 4.900 & 15.43 & 22.23 \\
\hline 10 & .9821 & 5.964 & 15.02 & 20.07 \\
\hline 18 & .9998 & 6.000 & 15.00 & 20.00 \\
\hline
\end{tabular}


The convergence is intermediate between the two previous cases, with the parameter $\sigma$ about .56 .

\section{Study of a K3 Surface}

3.1. Geometry. We now reach the heart of this article: the numerical study of a particular K3 surface $S$. This is the double cover of the plane branched over the sextic curve $x^{6}+y^{6}+z^{6}=0$. Thus $S$ is defined by the equation $w^{2}=x^{6}+y^{6}+z^{6}$, where $w$ is a point in the total space of the line bundle $O(3)$ over $\mathbf{C P}^{2}$. Most of the time we work in the affine piece of $S$ which is the subset of $\mathbf{C}^{3}$, with co-ordinates $(x, y, w)$, cut out by the equation $w^{2}=x^{6}+y^{6}+1$. We fix the nowhere-vanishing holomorphic 2-form $\theta$ on $S$ given in this affine piece by

$$
\theta=\frac{d x d y}{w}=\frac{d x d y}{\sqrt{x^{6}+y^{6}+1}}
$$

This determines a volume form $\nu=\theta \bar{\theta}$.

The surface $S$ has many symmetries. These are generated by

- The permutations of $x, y, z$

- Multiplication of $x, y$ by sixth roots of unity.

- The covering involution $w \mapsto-w$.

- The antiholomorphic involution given by complex conjugation of all coordinates.

Thus we get a symmetry group $\Gamma$ of order $6 \times 6^{2} \times 2 \times 2=864$, preserving the volume form $\nu$.

We now wish to evaluate the total mass

$$
\operatorname{Vol}(S, \nu)=\int_{S} \nu
$$

This is not strictly necessary for our main purpose, but gives a valuable check on the accuracy of our numerical calculations in the next section. We can evaluate the integral by exploiting the fact that $S$ is an an elliptic surface, of a very special kind. To see the elliptic fibration we consider the map $f:[x, y, z] \mapsto\left[x^{3}, y^{3}, z^{3}\right]$ from $\mathbf{P}^{2}$ to $\mathbf{P}^{2}$. This maps the sextic branch curve to the conic $X^{2}+Y^{2}+Z^{2}=0$. Thus the covering $S \rightarrow \mathbf{P}^{2}$ is the pull back by $f$ of the familiar covering of the quadric $Q$ over the plane, branched along the conic. Now $Q=\mathbf{P}^{1} \times \mathbf{P}^{1}$ is fibred by lines (in two different ways). These fibres are the preimages under the covering 
$Q \rightarrow \mathbf{P}^{2}$ of the lines tangent to the conic. The preimages of these lines under $f$ are cubic curves in the plane tangent to the sextic branch curve at each intersection point, and these are the elliptic curves whose lifts to the double cover yield an elliptic fibration of $S$.

To see all of this more explicitly we work in affine coordinates. Let $C$ be the plane curve with equation $p^{3}+q^{3}+1=0$. Recall that the conic $\lambda^{2}+\mu^{2}+1=0$ is parametrised by a rational variable $\tau$ with

$$
\lambda=\frac{1}{2 i}\left(\tau+\tau^{-1}\right), \quad \mu=\frac{1}{2}\left(\tau-\tau^{-1}\right) .
$$

Now for a point $(p, q)$ in the curve $C$ and a complex parameter $\tau$ set

$$
x=\frac{p}{\lambda^{1 / 3}} \quad y=\frac{q}{\mu^{1 / 3}},
$$

where $\lambda=\lambda(\tau), \mu=\mu(\tau)$ are given by Equation 1. Of course we have to deal with the cube roots in Equation 2, so initially we just regard $\tau$ as varying in an appropriate open set $\Omega$ in $\mathbf{P}^{1}$. A few lines of algebra show that if we set

$$
w=i \frac{p^{3}-\lambda^{2}}{\lambda \mu}
$$

then $x, y, w$ satisfy the equation $w^{2}=x^{6}+y^{6}+1$. In other words, we have defined a holomorphic isomorphism $F$ from $\Omega \times C \subset \mathbf{P}^{1} \times C$ to an open set in $S$. Further straightforward calculation shows that

$$
F^{*}(\theta)=\frac{2^{2 / 3}}{3} \phi \psi
$$

where

$$
\phi=\frac{d p}{\left(1-p^{3}\right)^{2 / 3}} \quad, \quad \psi=\frac{d \tau}{\left(\tau\left(\tau^{4}-1\right)\right)^{1 / 3}} .
$$

The form $\phi$ is just the standard holomorphic 1-form on the elliptic curve $C$. The form $\psi$ is initially defined only an open set $\Omega$ in $\mathbf{P}^{1}$. We could introduce a covering $\Sigma \rightarrow \mathbf{P}^{1}$ such that $\psi$ lifts to a holomorphic form on $\Sigma$, but there is no need to do this because the 2-form $\psi \wedge \bar{\psi}$ is a well defined integrable form on $\mathbf{P}^{1}$. Since we can cover a dense open set in $S$ by taking $\Omega$ to be a cut plane, we see that

where

$$
\operatorname{Vol}(S, \nu)=\frac{2^{4 / 3}}{9} I J
$$

$$
I=i \int_{C} \phi \wedge \bar{\phi}, \quad J=i \int_{\mathbf{P}^{1}} \psi \wedge \bar{\psi}
$$


Now let $W$ we the wedge-shaped region in $\mathbf{C}$ defined by $0<\arg (p)<\pi / 3$. A dense open set in the curve $C$ is covered by 18 copies of $W$ (6 rotations in $\mathbf{C}$ each of which has 3 lifts to $C$ ). So

$$
I=18 \int_{W} \phi \wedge \bar{\phi}
$$

Now it is easy to see that the indefinite integral of $\left(1-p^{3}\right)^{-2 / 3}$ maps $W$ to an equilateral triangle in $\mathbf{C}$ with side length $L_{I}$ where

$$
L_{I}=\int_{0}^{1} \frac{d p}{\left(1-p^{3}\right)^{2 / 3}} .
$$

It follows that

$$
I=18 \frac{\sqrt{3}}{2} L_{I}^{2}
$$

Similarly let $Q$ be the domain in $\mathbf{C}$ defined by the conditions $|\tau|<1,0<\arg (\tau)<$ $\pi / 2$. This is a fundamental domain for an action of a group of order 8 generated by $\tau \mapsto \tau^{-1}$ and $\tau \mapsto i \tau$ which preserves the form $\psi \wedge \bar{\psi}$. So

$$
J=8 \int_{Q} \psi \wedge \bar{\psi}
$$

The indefinite integral of $\psi$ maps $Q$ to another equilateral triangle with side length $L_{J}$ say, so

$$
J=8 \frac{\sqrt{3}}{2} L_{J}^{2},
$$

where

$$
L_{J}=\int_{0}^{1} \frac{d \tau}{\left(\tau\left(1-\tau^{4}\right)\right)^{1 / 3}} .
$$

Finally, the elementary substitution,

$$
p=\left(\frac{\tau^{2}-1}{\tau^{2}+1}\right)^{2 / 3}
$$

shows that

$$
L_{J}=3 \frac{4^{2 / 3}}{8} L_{I} .
$$

(We can also interpret the substitution of Equation 4 as defining a covering map from $\Sigma$ to $C$.) Putting everything together we obtain

$$
\operatorname{Vol}(S, \nu)=27 L_{I}^{4},
$$


where $L_{I}$ is the one-dimensional integral in Equation 3. The author evaluating this numerically to get $L_{I}=1.76664$, which yields

$$
\operatorname{Vol}(S, \nu)=263.00
$$

to 5 significant figures. Subsequently, Julien Keller pointed out that there is an exact formula

$$
\operatorname{Vol}(S, \nu)=\frac{3}{(2 \pi)^{4}} \Gamma(1 / 3)^{12}
$$

which gives the volume as 262.99941, to 8 significant figures.

3.1.1. Linear systems on $S$. We will study the general procedures of Section (2.2) for line bundles $O(k)$ over $S$ : powers of the lift of the hyperplane bundle $O(1)$ on $\mathbf{P}^{2}$. We will consider three cases, when $k=3,6,9$. The symmetry group of $S$ acts on $H^{0}(S ; O(k))$ and we can restrict attention to invariant metrics. It is a straightforward matter to describe these. There is a natural holomorphic section $w$ of $O(3)$ over $S$ and for $k \geq 3$ we have

$$
H^{0}(S ; O(k))=H^{0}\left(\mathbf{P}^{2}, O(k)\right) \oplus w H^{0}\left(\mathbf{P}^{2} ; O(k-3)\right) .
$$

The two summands are eigenspaces of the action of the covering involution, so must be orthogonal for any invariant metric. The sections of $O(j)$ over $\mathbf{P}^{2}$ are represented, in our affine piece, by linear combinations of monomials $x^{p} y^{q}$ for $p+q \leq j$. So we have a standard basis of the space labelled by the integer points in a triangle. We will draw this as a right angled triangle but it is better to think of it as an equilateral triangle (with a hexagonal lattice) which makes the action of the group of permutations of the projective coordinates $x, y, z$ apparent. Now considering the action of multiplication by sixth roots of unity we see that we can only have a nontrivial inner product between monomials $x^{p} y^{q}$ and $x^{r} y^{s}$ if $p \equiv r$ and $q \equiv s$ modulo 6 . These inner products must be real numbers, due to the symmetry under the antiholomorphic involution of $S$. 
Now for $k=3$ an invariant metric must be diagonal in our standard basis. The metric is specified by 4 real parameters $a_{I}, a_{I I}, a_{I I I}, b_{I}$ as indicated.

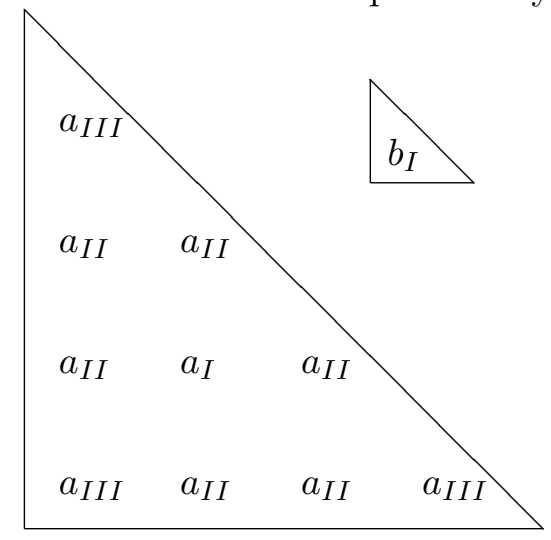

In other words, the function $D$ associated to the metric is given, in our affine coordinates, by

$D=a_{I}|x y|^{2}+a_{I I}\left(|x|^{2}+|x|^{4}+|y|^{2}+|y|^{4}+\left|x^{2} y\right|^{2}+\left|y^{2} x\right|^{2}\right)+a_{I I I}\left(|x|^{6}+|y|^{6}+1\right)+b_{I}|w|^{2}$

For $k=6$ we again have diagonal elements filling up two triangles, invariant under the permutations of $x, y, z$. This gives us 10 parameters $a_{I}, a_{I I}, \ldots a_{V I I}$, $b_{I}, b_{I I}, b_{I I I}$ according to the scheme indicated:

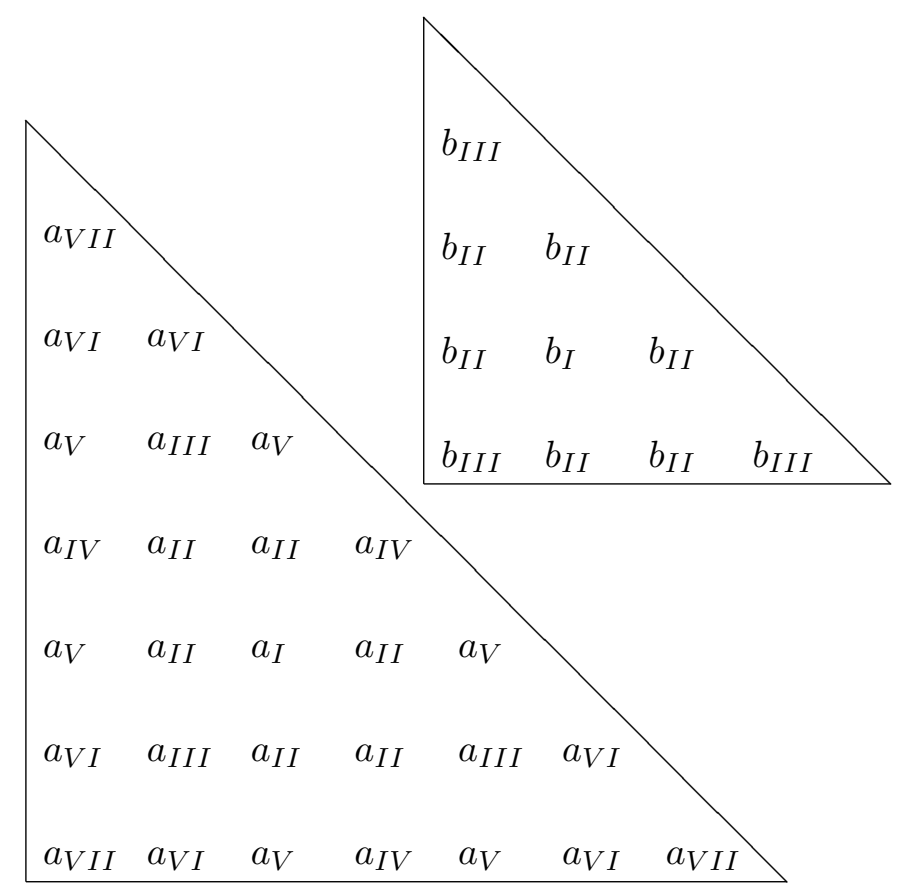


We also have three non-trivial off-diagonal terms corresponding to the inner products between $1, x^{6}, y^{6}$. These must all be equal, by symmetry, so we get one further real parameter $C$. In other words the matrix of our inner product contains a $3 \times 3$ block

$$
\left(\begin{array}{ccc}
a_{V I I} & C & C \\
C & a_{V I I} & C \\
C & C & a_{V I I}
\end{array}\right)
$$

(We could diagonalise this by using a different basis, but to fit in with the notation below we will not do so.)

In other words, the function $D$ is given by

$D=\sum a_{p, q}|x|^{2 p}|y|^{2 q}+C\left(x^{6} \bar{y}^{6}+\bar{x}^{6} y^{6}+x^{6}+\bar{x}^{6}+y^{6}+\bar{y}^{6}\right)+|w|^{2} \sum b_{p, q}|x|^{2 p}|y|^{2 q}$, where the coefficients $a_{p, q}, b_{p, q}$ are given by the parameters $a_{I}, \ldots, a_{V I I}, b_{I}, \ldots, b_{I I I}$ in the manner indicated above.

Finally we consider the case $k=9$. The diagonal elements in the big triangle are specified by twelve parameters $a_{I}, \ldots, a_{X I I}$ in the manner indicated (here we only draw a piece of the big triangle, the remainder follows by symmetry).

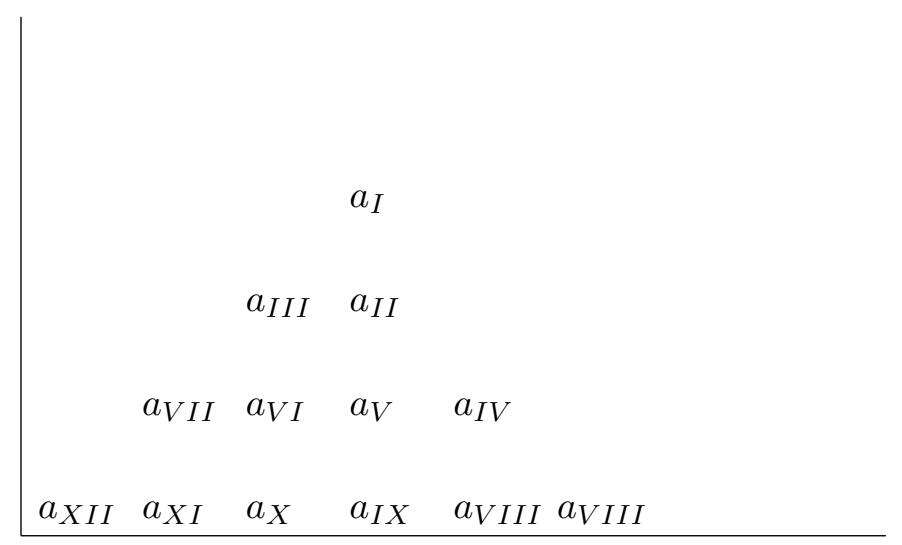

There are now more allowable off-diagonal elements, specified by 6 independent parameters $C_{1}, \ldots, C_{6}$. These fit into $3 \times 3$ blocks in the matrix of the metric of the following forms:

$$
\left(\begin{array}{ccc}
a_{V I} & C_{2} & C_{1} \\
C_{2} & a_{X} & C_{3} \\
C_{1} & C_{3} & a_{X I}
\end{array}\right),\left(\begin{array}{ccc}
a_{X I I} & C_{4} & C_{4} \\
C_{4} & a_{I X} & C_{5} \\
C_{4} & C_{5} & a_{I X}
\end{array}\right),\left(\begin{array}{ccc}
a_{V I I} & C_{6} & C_{6} \\
C_{6} & a_{V I I} & C_{6} \\
C_{6} & C_{6} & a_{V I I}
\end{array}\right)
$$


The discussion of the small triangle repeats that in the $k=6$ case above: we have 8 parameters $b_{I}, \ldots, b_{V I I}, C^{\prime}$.

To sum up, we have now specified how invariant metrics on $H^{0}(S ; O(k))$ are determined by 4,11 and 26 real parameters respectively in the three cases $k=$ $3,6,9$. Notice that these are vast reductions on the dimensions of the full spaces of metrics $\operatorname{dim} H^{0}(S ; O(k))^{2}$, which are 121, 1444,6889 respectively.

\subsection{Numerical results.}

3.2.1. Numerical volume. The numerical implementation of the algorithm of (2.2) for finding a balanced metric is completely specified by the choice of an approximation $\nu^{*}=\sum \nu_{i} \delta_{z_{i}}$ for the given volume form $\nu$ on $S$. That is, what we compute is the sequence $T_{\nu^{*}}^{r}\left(H_{0}\right)$. Our numerical results are obtained using a family of such approximations which depend on four integer parameters $n_{x}, n_{p}, n_{u}, n_{w}$ : the larger these parameters are the more points $z_{i}$ are used, which should give a better approximation, at the expense of extra computation. More details are given in the next section, suffice it to say here that $n_{x}, n_{p}$ determine the contribution from one chart and $n_{u}, n_{w}$ from another. We should also emphasise that our approximation exploits the invariance of the functions we need to integrate under the group of symmetries of $S$.

As a first test of our approximations we compute the volume $\operatorname{Vol}\left(S, \nu_{0}\right)$ of $S$ in the measure $\nu$. We obtain then a family of approximations $V\left(n_{x}, n_{p}, n_{u}, n_{w}\right)$ with

$$
V\left(n_{x}, n_{p}, n_{u}, n_{w}\right)=V_{1}\left(n_{x}, n_{p}\right)+V_{2}\left(n_{u}, n_{w}\right) .
$$

We find:

\begin{tabular}{|l||l|}
\hline$V_{1}(10,10)=265.84$ & $V_{2}(10,10)=6.5557$ \\
\hline$V_{1}(20,20)=256.61$ & $V_{2}(14,10)=6.3227$ \\
\hline$V_{1}(30,30)=256.88$ & $V_{2}(20,20)=6.2575$ \\
\hline$V_{1}(40,40)=256.70$ & $V_{2}(24,20)=6.2536$ \\
\hline
\end{tabular}

So, for example, $V(40,40,24,20)=256.70+6.25=262.95$ and $V(20,20,14,10)=262.93$. Recall that the theoretical analysis gave $\operatorname{Vol}(S, \nu)=$ 263.00. These figures suggest that, for values of the parameters similar to those 
above, we can integrate reasonably smooth functions on $S$ with an accuracy of about 3 or 4 significant figures.

3.2.2. The case $k=3$. We now implement our " $T_{\nu}$-algorithm" to find the $\nu$ balanced metric for the line bundle $O(3)$ on $S$. We choose, arbitrarily, the initial metric specified by the four parameters $(1,1,1,1)$. Then we obtain, using the approximating measure $\nu^{*}(20,20,14,10)$ :

\begin{tabular}{|l||l|l|l|l|}
\hline $\mathrm{r}$ & $a_{I}$ & $a_{I I}$ & $a_{I I I}$ & $b_{I}$ \\
\hline 0 & 1 & 1 & 1 & 1 \\
\hline 1 & 12.68 & 8.758 & 5.257 & 2.722 \\
\hline 2 & 13.20 & 8.807 & 4.987 & 2.439 \\
\hline 3 & 13.25 & 8.811 & 4.959 & 2.414 \\
\hline 4 & 13.26 & 8.812 & 4.956 & 2.412 \\
\hline 5 & 13.26 & 8.812 & 4.956 & 2.412 \\
\hline
\end{tabular}

(Recall that everything is preserved by rescaling the metrics. Here, and in the similar results to follow, we normalise the metrics for $r \geq 1$, up to scale, in an arbitrary way.) Rounding off to $4 \mathrm{SF}$, the iteration reaches a fixed point after four steps. Taking a finer approximation $\nu^{*}(30,30,20,14)$ makes hardly any change: to $4 \mathrm{SF}$, the fixed point is now $13.26,8.816,4.956,2.415$. So we have some confidence that these last values of the parameters give the balanced metric on $H^{0}(S ; O(3))$ to high accuracy.

We now examine the Fubini study volume form $\mu$ on $S$ determined by this balanced metric. (To fix constants, we take the Kahler form in the class $2 \pi c_{1}(O(3))$.) That is, for given parameters $n_{x}, n_{p}, n_{u}, n_{w}$ we compute the ratio $\mu / \nu$ at each of the points in the support of $\nu^{*}\left(n_{x}, n_{p}, n_{u}, n_{w}\right)$ (see Section (3.4.2) for more details of the calculation). Now we have another test of our numerical methods by integrating $\mu / \nu$ with respect to the measure $\nu^{*}\left(n_{x}, n_{p}, n_{u}, n_{w}\right)$. By Chern-Weil theory

$$
\int_{S} d \mu=9.2 \cdot 4 \cdot \pi^{2}=710.61
$$

so the ratio of the total volumes with respect to $\mu$ and $\nu$ is $710.61 / 263=2.7019$. Taking parameters $[28,28,20,14]$ we get

$$
\int_{S} \frac{\mu}{\nu} d \nu^{*}=2.7023 \int_{S} d \nu^{*}
$$


which is again a fair agreement. Set $\eta=(2.7019)^{-1} \mu / \nu$, so the mean value of $\eta$ with respect to $\nu$ is 1 and the deviation of $\eta$ from the constant function 1 is a measure of the difference between the balanced metric and the Calabi-Yau metric on $S$, normalised by the appropriate scale factor. We compute

- The maximum value of $\eta$,

- The minimum value of $\eta$,

- The mean value of $|\eta-1|$ with respect to $\nu$,

- The distribution function of $\eta$ with respect to $\nu$.

More precisely, of course, we compute the maximum and minimum values over the support of a $\nu^{*}\left(n_{x}, n_{p}, n_{u}, n_{w}\right)$, for appropriate parameter values, and we compute the mean and distribution function with respect to $\nu^{*}$. The result (with parameters $[28,28,20,14])$ is

$$
\max \eta=1.496, \quad \min \eta=.2501, \quad \operatorname{Mean}(|\eta-1|)=.262
$$

For the (approximate) distribution function we give in the first row of the table a collection of ranges and in the second row the percentages of the $\nu$-volume of $S$ where $\eta$ takes values in the given range.

\begin{tabular}{|l|l|l|l|l|l|l|l|l|}
\hline $.25-.4$ & $.4-.55$ & $.55-.7$ & $.7-.85$ & $.85-1$ & $1-1.15$ & $1.15-1.3$ & $1.3-1.45$ & $1.45-$ \\
\hline 3.4 & 6.9 & 9.6 & 11.7 & 15.3 & 17.3 & 15.0 & 15.8 & 5.1 \\
\hline
\end{tabular}

We see that $\mu$ is not really a good approximation to $2.7019 \nu$, as we would expect with this very low value of $k$. So we do not proceed to try to find a "refined approximation" in this case.

3.2.3. The case $k=6$. We carry through the same procedure as in the previous subsection. With parameters $[20,20,14,10]$ the following table shows the convergence to the balanced metric. 


\begin{tabular}{|l||l|l|l|l|l|l|l|l|l|l|l|}
\hline $\mathrm{r}$ & $a_{I}$ & $a_{I I}$ & $a_{I I I}$ & $a_{I V}$ & $a_{V}$ & $a_{V I}$ & $a_{V I I}$ & $b_{I}$ & $b_{I I}$ & $b_{I I I}$ & $C$ \\
\hline 0 & 1 & 1 & 1 & 1 & 1 & 1 & 1 & 1 & 1 & 1 & 0 \\
\hline 1 & 51.7 & 43.3 & 33.1 & 23.9 & 21.8 & 15.9 & 7.55 & 21.8 & 14.1 & 7.37 & .915 \\
\hline 2 & 56.4 & 45.9 & 33.6 & 23.2 & 20.8 & 14.3 & 5.85 & 20.3 & 12.2 & 5.66 & .509 \\
\hline 3 & 57.6 & 46.5 & 33.7 & 23.0 & 20.6 & 13.9 & 5.47 & 19.9 & 11.7 & 5.28 & .432 \\
\hline 4 & 57.6 & 46.6 & 33.7 & 23.0 & 20.6 & 13.9 & 5.46 & 19.9 & 11.7 & 5.27 & .430 \\
\hline$\infty$ & 57.6 & 46.6 & 33.7 & 23.0 & 20.6 & 13.9 & 5.45 & 19.9 & 11.7 & 5.26 & .429 \\
\hline
\end{tabular}

Analysis of the convergence suggests that the parameter $\sigma$ is about .22. Increasing the numerical integration parameters to $[30,30,20,16]$ gives a fixed point, to 4 significant figures,

\begin{tabular}{|l|l|l|l|l|l|l|l|l|l|l|}
\hline 57.70 & 46.61 & 33.67 & 23.01 & 20.56 & 13.86 & 5.453 & 19.95 & 11.70 & 5.267 & .4324 \\
\hline
\end{tabular}

We take these parameters as our numerical balanced metric.

The volume form of the balanced metric yields a function $\eta$ with:

$$
\max \eta=1.065, \min \eta=.679, \operatorname{Mean}(|\eta-1|)=.058 .
$$

The distribution function of $\eta$ is given by the following table, in which again the first row gives the range and the second gives the percentage of the total volume lying within the range.

\begin{tabular}{|l|l|l|l|l|l|l|l|l|}
\hline-.7 & $.7-.75$ & $.75-.8$ & $.8-.85$ & $.85-.9$ & $.9-.95$ & $.95-1$ & $1-1.05$ & $1.05-$ \\
\hline .07 & 1.2 & 2.2 & 3.3 & 4.9 & 7.9 & 14.0 & 35.0 & 31.4 \\
\hline
\end{tabular}

This is a much better approximation to the Calabi-Yau metric. The function $\eta$ is close to 1 over most of the manifold; further exploration shows the set where it deviates substantially from 1 is a neighbourhood of the branch curve of the double cover, on which $\eta$ is small.

We now move on to search for a refined approximation, using the algorithm described in (2.2.1) above. Thus we compute the tensor $\pi(\eta-1) \in H\left(L^{k}\right)$ as a measure of the size of the error term. If we work with an orthonormal basis of sections then the individual matrix entries $\eta_{\alpha \beta}$ can be interpreted as analogues of Fourier coefficients of $\eta$. For simplicity we work instead with an approximately orthonormal basis given by rescaling the standard monomials, which we justify 
by the fact that the off-diagonal term $C$ is relatively small and we are working close to the balanced metric. Thus for any metric we define

$$
\begin{aligned}
& \eta(p q)=R a_{p q} \int_{S}(\eta-1) \frac{|x|^{2 p}|y|^{2 q}}{D} d \nu, \\
& \bar{\eta}(p q)=R b_{p, q} \int_{S}(\eta-1) \frac{\left.|x|^{2 p}\left|y^{2 p}\right| w\right|^{2}}{D}
\end{aligned}
$$

and

$$
\eta_{C}=R a_{0,0} \int_{S}(\eta-1) \frac{\operatorname{Re}\left(x^{6}\right)}{D} d \nu_{0}
$$

where $R=\operatorname{dim} H^{0}\left(L^{k}\right) / \operatorname{Vol}\left(S, \nu_{0}\right)=38 / 263$. The terms $\eta(p q), \bar{\eta}(p q)$ have the same symmetries as the coefficients so we can extract 11 different terms $\eta_{I}, \ldots, \eta_{V I I}$, $\bar{\eta}_{I}, \ldots, \bar{\eta}_{I I I}, \eta_{C}$ corresponding to the coefficients $a_{I} \ldots, b_{I}, \ldots, C$. As described in Section 2, the diagonal terms $\eta_{I}, \ldots, \bar{\eta}_{I I I}$ can be interpreted approximately as weighted averages of the error $\eta-1$. (The approximations we are making here only involve the interpretetation of the data, not the actual algorithms.)

The next two tables display the first 5 steps of the refining procedure with the parameter $\kappa$ equal to 2.5 . The sequence of metrics is:

\begin{tabular}{|l||l|l|l|l|l|l|l|l|l|l|l|}
\hline $\mathrm{r}$ & $a_{I}$ & $a_{I I}$ & $a_{I I I}$ & $a_{I V}$ & $a_{V}$ & $a_{V I}$ & $a_{V I I}$ & $b_{I}$ & $b_{I I}$ & $b_{I I I}$ & $C$ \\
\hline 0 & 57.70 & 46.61 & 33.67 & 23.01 & 20.56 & 13.86 & 5.452 & 19.95 & 11.70 & 5.267 & .4326 \\
\hline 1 & 53.63 & 44.15 & 33.47 & 23.92 & 21.62 & 15.08 & 6.380 & 21.73 & 13.19 & 6.124 & .6059 \\
\hline 2 & 51.62 & 43.72 & 33.51 & 24.18 & 21.88 & 15.26 & 6.394 & 22.52 & 13.55 & 6.136 & .6100 \\
\hline 3 & 51.01 & 43.50 & 33.59 & 24.34 & 22.03 & 15.34 & 6.347 & 23.17 & 13.74 & 6.096 & .5949 \\
\hline 4 & 50.57 & 43.35 & 33.62 & 24.44 & 22.13 & 15.39 & 6.310 & 23.61 & 13.87 & 6.066 & .5785 \\
\hline 5 & 50.22 & 43.24 & 33.74 & 24.51 & 22.19 & 15.43 & 6.284 & 24.03 & 13.95 & 6.047 & .5621 \\
\hline
\end{tabular}

The corresponding sequence of $\eta$-coefficients, multiplied by $10^{3}$ :

\begin{tabular}{|l||l|l|l|l|l|l|l|l|l|l|l|}
\hline $\mathrm{r}$ & $\eta_{I}$ & $\eta_{I I}$ & $\eta_{I I I}$ & $\eta_{I V}$ & $\eta_{V}$ & $\eta_{V I}$ & $\eta_{V I I}$ & $\bar{\eta}_{I}$ & $\bar{\eta}_{I I}$ & $\bar{\eta}_{I I I}$ & $\eta_{C}$ \\
\hline 0 & -56.1 & -42.9 & -25.2 & -8.0 & -3.6 & 10.1 & 32.0 & 10.7 & 25.1 & 38.3 & 3.0 \\
\hline 1 & -10.7 & -6.9 & -2.6 & 1.2 & 1.6 & 1.7 & -4.8 & 11.2 & 7.6 & -2.2 & .3 \\
\hline 2 & -5.9 & -3.3 & -.4 & 1.3 & 1.4 & .8 & -5.1 & 9.3 & 4.4 & -3.9 & -.2 \\
\hline 3 & -4.4 & -2.3 & .03 & .8 & .9 & .5 & -3.3 & 7.7 & 2.8 & -2.8 & -.4 \\
\hline 4 & -3.4 & -1.6 & .2 & .5 & .6 & .3 & -1.8 & 6.4 & 1.7 & -1.9 & -.6 \\
\hline 5 & -2.8 & -1.3 & .4 & .3 & .3 & .2 & -.8 & 5.4 & .9 & -1.3 & -.7 \\
\hline
\end{tabular}


The size of the error terms behaves as follows:

\begin{tabular}{|l||l|l|l|}
\hline $\mathrm{r}$ & Max. & Min. & Mean Error(\%) \\
\hline 0 & 1.065 & .679 & $5.80 \%$ \\
\hline 1 & 1.046 & .804 & $2.61 \%$ \\
\hline 2 & 1.043 & .830 & $2.21 \%$ \\
\hline 3 & 1.041 & .843 & $1.94 \%$ \\
\hline 4 & 1.039 & .853 & $1.79 \%$ \\
\hline 5 & 1.05 & .860 & $1.75 \%$ \\
\hline
\end{tabular}

We see that for the first four steps the error term does decrease, according to any of the measures above. The initial rate of decrease is large but this soon slows down. At the fifth step the maximum value increases, although the mean and minimum improve. The most obvious phenomenon is that the coefficient $a_{I}$ is decreased, along with the "nearby" co-efficients in the big triangle, and the co-efficient $b_{I}$ is increased, along with the nearby coefficients in the small triangle. This has the effect of increasing the volume form $\mu$ near the branch curve, and so compensating for the deviation in the balanced metric. We repeat the process many times, with various values of $\kappa$. After a while the maximum value decreases again, and for example we achieve at an intermediate stage the following metric, which we call $\omega_{6}^{\prime}$ :

\begin{tabular}{|l|l|l|l|l|l|l|l|l|l|l|}
\hline 46.61 & 42.69 & 34.94 & 25.04 & 22.56 & 15.34 & 6.301 & 28.95 & 13.79 & 6.130 & .2854 \\
\hline
\end{tabular}

This has

$$
\operatorname{Max} \eta=1.031, \operatorname{Min} \eta=.898, \operatorname{Mean}(|\eta-1|)=.017,
$$

and $\eta$-coefficients, multiplied by $10^{3}$ :

$$
\begin{array}{|l|l|l|l|l|l|l|l|l|l|l|}
\hline-.57 & -.11 & .1 & .11 & .04 & -.3 & .25 & .9 & -.2 & .1 & -.2 \\
\hline
\end{array}
$$

The distribution function is:

\begin{tabular}{|l|l|l|l|l|l|l|l|l|}
\hline-.925 & $.925-.94$ & $.94-.955$ & $.955-.97$ & $.97-.985$ & $.985-1$ & $1-1.015$ & $1.015-1.03$ & $1.03-$ \\
\hline .32 & .45 & 1.0 & 1.4 & 7.9 & 38.8 & 33.4 & 16.7 & .004 \\
\hline
\end{tabular}

We contine the process still further. The rate of change becomes extremely slow, and we finally give up at a metric we will call $\omega_{6}^{\prime \prime}$ defined by metric coefficients

\begin{tabular}{|l|l|l|l|l|l|l|l|l|l|l|}
\hline 43.34 & 42.77 & 35.84 & 25.31 & 22.86 & 14.88 & 6.362 & 30.19 & 13.78 & 6.207 & .1341 \\
\hline
\end{tabular}


Which has

$$
\operatorname{Max} \eta=1.050, \operatorname{Min} \eta=.909, \operatorname{Mean}(|\eta-1|)=.0111
$$

and $\eta$-coefficients, multiplied by $10^{3}$ :

\begin{tabular}{|l|l|l|l|l|l|l|l|l|l|l|}
\hline-.21 & .011 & .063 & -.026 & .020 & -.070 & -.004 & -.063 & .024 & .005 & -.047 \\
\hline
\end{tabular}

We take this metric $\omega_{6}^{\prime \prime}$ as our best estimate for a refined approximation. Now there are several points to make. First, the $\eta$-coefficients for $\omega_{6}^{\prime \prime}$ are much smaller than those for the balanced metric $\omega_{6}$, by a factor of more than 100 . However the very slow movement under our primitive algorithm means that it is not completely clear that there is a genuine, exact, refined approximation close to $\omega_{6}^{\prime \prime}$. Second, the metric $\omega_{6}^{\prime \prime}$ is probably not significantly better as an approximation to the Calabi-Yau metric than the intermediate metric $\omega_{6}^{\prime}$, and could well be worse. So our overall conclusion is that the ideas we developed in (2.2.1) are effective, in this case, in generating some much better approximations than the balanced metric (such as $\omega_{6}^{\prime}$ or even the fourth step in the process), although a more exact and sophisticated analysis of the whole situation is required.

3.3. The case $k=9$. The numerical results in this case follow very much the same pattern as for $k=6$ above, so we will be brief. The crucial difference, of course, is that the error terms are smaller. Recall that we now have 26 parameters which we display in four-row form as

\begin{tabular}{|l|l|l|l|l|l|l|}
\hline$a_{I}$ & $a_{I I}$ & $a_{I I I}$ & $a_{I V}$ & $a_{V}$ & $a_{V I}$ & $a_{V I I}$ \\
\hline$a_{V I I I}$ & $a_{I X}$ & $a_{X}$ & $a_{X I}$ & $a_{X I I}$ & & \\
\hline$b_{I}$ & $b_{I I}$ & $b_{I I I}$ & $b_{I V}$ & $b_{V}$ & $b_{V I}$ & $b_{V I I}$ \\
\hline$C_{1}$ & $C_{2}$ & $C_{3}$ & $C_{4}$ & $C_{5}$ & $C_{6}$ & $C^{\prime}$ \\
\hline
\end{tabular}

We find the balanced metric is:

\begin{tabular}{|l|l|l|l|l|l|l|}
\hline 992.0 & 858.9 & 682.7 & 572.8 & 525.7 & 401.8 & 227.6 \\
\hline 200.8 & 176.9 & 130.3 & 69.46 & 19.60 & & \\
\hline 460.1 & 346.2 & 218.2 & 147.8 & 123.8 & 68.75 & 19.57 \\
\hline 18.45 & 19.26 & 17.82 & 7.275 & 8.245 & 33.21 & .1903 \\
\hline
\end{tabular}

The convergence parameter $\sigma$ is about .33. The balanced metric has

$$
\operatorname{Max} \eta=1.024, \operatorname{Min} \eta=.833, \operatorname{Mean}(|\eta-1|)=.025 \text {. }
$$


The largest modulus of the $\eta$-coefficients is about $34 \times 10^{-3}$.

The distribution function is

\begin{tabular}{|l|l|l|l|l|l|l|l|l|}
\hline-.88 & $.88-.9$ & $.9-.92$ & $.92-.94$ & $.94-.96$ & $.96-.98$ & $.98-1$ & $1-1.02$ & $1.02-$ \\
\hline 1.3 & 1.7 & 2.1 & 3.1 & 4.2 & 6.6 & 12.1 & 29.9 & 39.1 \\
\hline
\end{tabular}

Thus over about $70 \%$ of the volume, $\eta$ lies in the range $1-1.025$. We carry out the refining procedure as before. The process seems to work better than for $k=6$, in that the error decreases steadily and our best estimate for the refined approximation yields the best approximation to the Calabi-Yau metric. This approximation, $\omega_{9}^{\prime}$ is

\begin{tabular}{|l|l|l|l|l|l|l|}
\hline 875.6 & 798.5 & 665.8 & 584.5 & 539.3 & 419.5 & 239.8 \\
\hline 214.3 & 190.4 & 140.3 & 76.43 & 21.76 & & \\
\hline 540.5 & 386.8 & 233.3 & 161.8 & 134.9 & 75.53 & 21.76 \\
\hline 19.89 & 25.89 & 19.57 & 8.406 & 14.44 & 31.69 & .5101 \\
\hline
\end{tabular}

The $\eta$-coefficients (times $10^{3}$ ) are

\begin{tabular}{|l|l|l|l|l|l|l|}
\hline-.09 & -.04 & -.001 & -.02 & -.02 & -.03 & -.05 \\
\hline-.03 & -.04 & -.03 & .005 & -.06 & & \\
\hline .16 & -.002 & -.08 & -.02 & -.02 & .01 & -.04 \\
\hline-.01 & .02 & .03 & .03 & .04 & -.02 & .03 \\
\hline
\end{tabular}

The error has

$$
\max \eta=1.009, \min \eta=.972, \operatorname{Mean}(|\eta-1|)=.0022,
$$

and distribution function:

\begin{tabular}{|l|l|l|l|l|l|l|l|}
\hline-.975 & $.975-.98$ & $.98-.985$ & $.985-.99$ & $.99-.995$ & $.995-1$ & $1-1.005$ & $1.005-$ \\
\hline .01 & .1 & .3 & .9 & 1.5 & 46.6 & 46.1 & 4.5 \\
\hline
\end{tabular}

So over more than $90 \%$ of the manifold $\eta$ is within $.5 \%$ of 1 . Notice that, in passing from $k=6$ to $k=9$, we reduce the mean error in the balanced metrics by a factor of about 2 , which is roughly $(9 / 6)^{2}$. On the other hand we reduce the mean error in the refined approximations by a factor of about 5 . This gives some 
small support for the idea that the refined approximations should yield rapid convergence, in $k$, to Calabi-Yau metrics.

This concludes our account of the main numerical results of this article. Notice that the function $|\theta|_{\omega}$ discussed in the Introduction is essentially $\eta^{-1 / 2}$, so the deviation from 1 will be about half that of $\eta$.

\subsection{Computational details.}

3.4.1. Charts. To integrate numerically over $S$ we need an appropriate system of coordinate charts, and the choice of good charts is an interesting geometrical question. Suppose $(\xi, \eta)$ are local holomorphic coordinates for some chart in an atlas, i.e. with $(\xi, \eta)$ lying in some bounded domain $D \subset \mathbf{C}^{2}$ and with the holomorphic 2-form given by $\theta=F(\xi, \eta) d \xi d \eta$. Then we want to realise three desireable properties:

- The oscillation of the function $F$ is not too large on $D$.

- It is easy to recognize if a point $(\xi, \eta)$ lies in $D$.

- The overlaps of this chart with the other charts in the atlas are not too small.

The last property is needed because our integration procedure will involve a partition of unity subordinate to the cover, so we need a smooth cut-off function $\psi_{D}$ supported in $D$ and equal to 1 outside the region corresponding to the other charts. The contribution from $D$ to the integral of a function $f$ on $S$ will have the shape

$$
\int_{D} f \psi_{D}|F|^{2} d \xi d \bar{\xi} d \eta d \bar{\eta}
$$

If the overlaps are small the derivative of $\psi_{D}$ will be large and the numerical integration will not be accurate.

As a first step we restrict to the open set $U$ in $S$ defined by the conditions

$$
|x|<1.2|z|,|y|<1.2|z|,
$$

in terms of the homogeneous co-ordinates $(x, y, z)$ on $\mathbf{C P}^{2}$. Clearly $S$ is covered by $U$ and its two images under the permutation action, so to integrate a $\Gamma$ invariant function it suffices to work in $U$. Then we can pass to affine coordinates, where $z=1$ and $U$ is represented as the polydisc $|x|,|y|<1.2$. Now let $U_{1}$ be the complement in $U$ of the product of the disc $|y|<1.2$ with suitable small 
neighbourhoods of the points where $x^{6}=-1$, chosen so that the roots of $\left(1+x^{6}\right)$ can be defined on $U_{1}$. Then

$$
w^{2}=\left(1+x^{6}\right)\left(1+\left(\frac{y}{\left(1+x^{6}\right)^{1 / 6}}\right)^{6}\right) .
$$

Set $q=\frac{w}{\sqrt{1+x^{6}}}$ so that

$$
q^{2}-1=\frac{y^{6}}{1+x^{6}}
$$

Now let $U_{2} \subset U_{1}$ be the region defined by the condition $\operatorname{Re}(q)>-1 / 2$. Then $U_{1}$ is covered by $U_{2}$ and its image under the covering involution (which takes $q$ to $-q)$. On $U_{2}$ we write $q=1+p^{6}$ so

$$
y=p\left(2+p^{6}\right)^{1 / 6}\left(1+x^{6}\right)^{1 / 6},
$$

where the root $\left(2+p^{6}\right)^{1 / 6}$ is well-defined on $U_{2}$ since we have cut out the zero where $q=-1$ and $2+p^{6}=0$. The upshot is that we have an open set $U_{2} \subset S$ on which we can take $x$ and $p$ as complex co-odinates, with $y$ given by Equation 5 and

$$
w=\left(1+p^{6}\right) \sqrt{1+x^{6}} .
$$

This gives one chart, which we call the "big chart" in $S$. The holomorphic form in these coordinates is

$$
\frac{2}{\left(2+p^{6}\right)^{5 / 6}\left(1+x^{6}\right)^{1 / 3}} d p d x
$$

Interchanging $x$ and $y$ takes $U_{1}$ to another open set $U_{1}^{\prime} \subset U$ say. The union $U_{1} \cup U_{1}^{\prime}$ covers all of $U$ save for small neighbourhoods of the points where $x^{6}=-1$ and $y^{6}=-1$. We want to define coordinates on an open set $V$ in $S$ covering a neighbourhood of the point $x=y=e^{i \pi / 6}$. Then $U$ will be covered by $U_{1}, U_{1}^{\prime}$ and the 36 images of $V$ under the action of multiplication by sixth roots of unity. To integrate a $\Gamma$-invariant function it will suffice to work in $U_{2}$ and $V$, provided we use suitable invariant cut-off functions and take due account of multiple counting by the symmetry group.

On $V$ we set $u=x^{6}-y^{6}$ and take $u$ and $w$ as local coordinates so

$$
x=\left(\frac{w^{2}+u-1}{2}\right)^{1 / 6}, y=\left(\frac{w^{2}-u-1}{2}\right)^{1 / 6} .
$$


This gives our other chart, the "small chart", on $S$, in which the holomorphic form is

$$
\frac{d u d w}{36 x^{5} y^{5}}
$$

The tension set up by the requirement discussed above for the two charts can now be seen as follows. In the small chart we need $|x|$ not to be too small and in the large chart we need $1+x^{6}$ not to be too small, but the two charts must have substantial overlap. We balance these requirements by defining the domain of the big chart to be the region where $\operatorname{Re}\left(x^{6}\right)>-(.9)^{6}=-.531$ and the domain of the small chart to be where $\operatorname{Re}\left(x^{6}\right)<-(.7)^{6}=-.117$. Then in the big chart $\left|1+x^{6}\right|>.47$, and in the small chart $|x|>.7$. The cut-off function has derivative (with respect to $x$ ) about $2(.9-.7)^{-1}=10$.

To integrate numerically in the big chart we use hexagonal lattices in the complex $x$ and $p$ variables. These allow us to take exact account of the residual $\mathbf{Z} / 6 \times \mathbf{Z} / 6$ symmetry (that is the integrands are functions of $x^{6}$ and $p^{6}$ ) saving a factor of nearly 36 in the calculation. We nearly double this factor using the further symmetry under complex conjugation. The lattice spacings are proportional to $n_{x}^{-1}, n_{p}^{-1}$ where $n_{x}, n_{p}$ are integer parameters mentioned in (3.2.1). To integrate numerically in the small chart we use square lattices in the $u$ and $w$ variables, taking advantage of the residual symmetry under complex conjugation and $w \mapsto-w$. The lattice spacings are proportional to $n_{u}^{-1}, n_{w}^{-1}$. In both charts the precise domains of integration are moderately complicated, and so the exact number of points where the functions are evaluated to approximate the integrals is not given by a simple formula. Writing $N_{1}\left(n_{x}, n_{p}\right)$ for the number in the big chart and $N_{2}\left(n_{u}, n_{w}\right)$ in the small chart, we have for example

$$
\begin{gathered}
N_{1}(10,10)=746, N_{1}(20,20)=9644, N_{1}(30,30)=45481 \\
N_{2}(10,10)=9737, N_{2}(20,20)=128149, N_{2}(24,20)=180435 .
\end{gathered}
$$

3.4.2. The volume form. To evaluate the volume form of the Fubini-Study metric defined by a given collection of co-efficients we work in the co-ordinates $x, y$. Let $V_{+}$be the complex vector space of dimension $(k+1)(k+2) / 2$ with a basis labelled by the integer points in the "big triangle" and $V_{-}$the space of dimension $(k-2)(k-1) / 2$ corresponding to the small triangle. Our data gives Hermitian metrics $\langle,\rangle_{ \pm}$on $V_{ \pm}$and so a Hermitian metric $\langle$,$\rangle on V_{+} \oplus V_{-}$. Let $r(x, y)=$ $r_{+}(x, y) \oplus r_{-}(x, y)$ be the vector valued function with the entries of $r_{+}(x, y)$ equal 
to $x^{p} y^{q}$ and the entries of $r_{-}(x, y)$ equal to $w x^{p} y^{q}$, where $w=\sqrt{1+x^{6}+y^{6}}$. (Of course $w$ is only defined up to a sign, but we choose a branch of the square root locally.) Write $r_{x}, r_{y}$ for the derivatives of $r$ with respect to $x, y$. Then away from the branch curve $w=0$ the ratio $V=d \mu_{F S} / d \nu_{0}$ of the Fubini-Study form and the fixed volume form $\theta \wedge \bar{\theta}$ is given by the formula

$$
V=\frac{|w|^{2}}{\|r\|^{6}} \operatorname{det}\left(\begin{array}{ccc}
\langle r, r\rangle & \left\langle r, r_{x}\right\rangle & \left\langle r, r_{y}\right\rangle \\
\left\langle r_{x}, r\right\rangle & \left\langle r_{x}, r_{x}\right\rangle & \left\langle r_{x}, r_{y}\right\rangle \\
\left\langle r_{y}, r\right\rangle & \left\langle r_{y}, r_{x}\right\rangle & \left\langle r_{y}, r_{y}\right\rangle
\end{array}\right)
$$

Of course this is the same whichever branch of the square root we take and the calculation is completely straightforward. The disadvantage is that we cannot use this formula near the branch curve because the individual terms in the determinant blow up (although in practice the formula seems to be accurate close enough to the branch curve for most purposes). A better formula is as follows. Let $v_{ \pm}$be the vector-valued functions, taking values in $V_{ \pm}$with entries $x^{p} y^{q}$. Thus $r_{+}=v_{+}$and $r_{-}=w v_{-}$. Put

$$
\delta_{x}=\left(v_{x}^{+}, w v_{x}^{-}\right), \delta_{y}=\left(v_{y}^{+}, w v_{y}^{-}\right)
$$

where subscripts denote differentiation. Set

$$
\hat{\delta_{x}}=\delta_{x}-\frac{\left\langle\delta_{x}, r\right\rangle}{\|r\|^{2}} \quad, \quad \hat{\delta_{y}}=\delta_{y}-\frac{\left\langle\delta_{y}, r\right\rangle}{\|r\|^{2}}
$$

Now write

$$
f_{x}=3 x^{5}, f_{y}=3 y^{5}
$$

Define

$$
Q_{x}=\frac{1}{\|r\|^{2}}\left(\left\langle\left\|v_{+}\right\|_{+}^{2}\left\langle v_{x}^{-}, v^{-}\right\rangle_{-}-\left\|v_{-}\right\|_{-}^{2}\left\langle v_{x}^{+}, v^{+}\right\rangle_{+}\right),\right.
$$

and $Q_{y}$ symmetrically. Then

$$
V=\frac{1}{\|r\|^{4}}\left(V_{1}+V_{2}-V_{3}+2 V_{4}-2 V_{5}\right)
$$


where

$$
\begin{aligned}
& V_{1}=|w|^{2}\left(\left\|\hat{\delta_{x}}\right\|^{2}\left\|\hat{\delta_{y}}\right\|^{2}-\left|\left\langle\hat{\delta_{x}}, \hat{\delta_{y}}\right\rangle\right|^{2}\right) ; \\
& V_{2}=\frac{\left\|v_{+}\right\|\left\|_{+}^{2}\right\| v_{-} \|_{-}^{2}}{\|r\|^{2}}\left\|f_{x} \hat{\delta_{y}}-f_{y} \hat{\delta_{x}}\right\|^{2} ; \\
& V_{3}=|w|^{2}\left|f_{x} Q_{y}-f_{y} Q_{x}\right|^{2} ; \\
& V_{4}=\operatorname{Re}\left(\left\|\hat{\delta_{y}}\right\|^{2} \overline{Q_{x}} \bar{w}^{2} f_{x}+\left\|\hat{\delta_{x}}\right\|^{2} \overline{Q_{y}} \bar{w}^{2} f_{y}\right) ; \\
& V_{5}=\operatorname{Re}\left(\left\langle\hat{\delta_{x}}, \hat{\delta_{y}}\right\rangle\left(\overline{f_{x}} Q_{y} w^{2}+f_{y} \overline{Q_{x}} \bar{w}^{2}\right)\right) .
\end{aligned}
$$

While it appears more complicated, this expression has advantages over Equation 6 even away from the branch curve.

\section{The Bergman Kernel}

Return to the general picture where $L \rightarrow X$ is an ample line bundle with a Hermitian metric and $d \nu$ is a fixed volume form on $X$. Then the space of sections $H^{0}\left(L^{k}\right)$ has an $L^{2}$ Hermitian inner product. For a point $x \in X$ we have an evaluation map

$$
e_{x}: H^{0}(L) \rightarrow L_{x},
$$

which is represented by the inner product, so there is an element $\sigma_{x} \in H^{0}(L) \otimes \overline{L_{x}}$ such that

$$
s(x)=\left\langle s, \sigma_{x}\right\rangle,
$$

for any section $s \in H^{0}(L)$. For $x, y \in X$ we define

$$
K(x, y)=\left|\sigma_{x}(y)\right|^{2},
$$

and the associated integral operator

$$
Q_{K}(f)(x)=R \int_{X} K(x, y) f(y) d \nu_{y}
$$

where $R=\operatorname{dim}\left(H^{0}\left(L^{k}\right) / \operatorname{Vol}(X, \nu)\right.$. (This factor is included to make $Q_{K}$ independent of scalings of $\nu$, and when the metric is balanced $Q_{K}(1)=1$.) Let $s_{\alpha}$ be an orthonormal basis of $H^{0}\left(L^{k}\right)$ with respect to the $L^{2}$ inner product. Then

$$
\sigma_{x}(y)=\sum_{\alpha} s_{\alpha}(y) \otimes \overline{s_{\alpha}(x)}
$$

and if we write

$$
f_{\alpha \beta}=\left(s_{\alpha}, s_{\beta}\right)
$$


(the pointwise inner product over $X$ ), we have

$$
K(x, y)=\sum_{\alpha, \beta} f_{\alpha \beta}(x) f_{\beta \alpha}(y) .
$$

Thus $Q_{K}$ is a finite-rank operator whose image lies in the finite-dimensional space $V \subset C^{\infty}(X)$ spanned by the $f_{\alpha \beta}$,i.e. the image $\iota\left(H\left(L^{k}\right)\right.$ in the notation of Section 2.2.1. The restriction of $Q_{K}$ to $V$ gives an endomorphism of $V$ with

$$
Q_{K}\left(\sum a_{\gamma \delta} f_{\gamma \delta}\right)=R \sum a_{\gamma \delta}\left\langle f_{\gamma \delta}, f_{\alpha \beta}\right\rangle f_{\alpha \beta}
$$

where $\langle$,$\rangle denotes the L^{2}$ inner product on functions. In other words we can define an endomorphism $Q: H\left(L^{k}\right) \rightarrow H\left(L^{k}\right)$ with matrix

$$
Q_{\alpha \beta, \gamma \delta}=R\left\langle f_{\gamma \delta}, f_{\alpha \beta}\right\rangle,
$$

and $\iota \circ Q=Q_{K} \circ \iota$. One interpretation of $Q$ is that it compares the two natural inner products on $V$. If we have a given metric on $H^{0}\left(L^{k}\right)$ then we can identify $H\left(L^{k}\right)$ with the self-adjoint endomorphisms of $H^{0}\left(L^{k}\right)$ and as such we have a standard Hilbert-Schmidt norm on $H\left(L^{k}\right)$ given by

$$
\left\|\left(a_{\alpha \beta}\right)\right\|_{H S}^{2}=\sum\left|a_{\alpha \beta}\right|^{2} .
$$

On $V$ we have the restriction of the $L^{2}$ norm and these are related by

$$
\|\iota(a)\|_{L^{2}}^{2}=R\langle a, Q(a)\rangle_{H S} .
$$

Notice that, if we regard $H\left(L^{k}\right)$ as the self-adjoint endomorphisms of $H^{0}\left(L^{k}\right)$ then $Q$ has been normalised so that, when the metric is balanced, $Q(1)=1$.

To illustrate these ideas, take $L \rightarrow X$ to be the line bundle $O(1)$ over $\mathbf{C P}^{1}$ with the standard metric, and standard area form. As in Section (2.1.1) we take the usual $S^{1}$ action on $\mathbf{C P}^{1}$ and restrict attention to the invariant part of $V$, which corresponds to the diagonal matrices in $H\left(L^{k}\right)$. With this restriction $Q$ is represented by a $(k+1) \times(k+1)$ matrix with entries

$$
Q_{i j}=\frac{k+1}{2 k+1} \frac{\left(\begin{array}{c}
k \\
i
\end{array}\right)\left(\begin{array}{l}
k \\
j
\end{array}\right)}{\left(\begin{array}{c}
2 k \\
i+j
\end{array}\right)} 0 \leq i, j \leq k
$$

(see the discussion in (4.3) below). 
4.1. The linearisation of the algorithm. One way in which the discussion above enters into our theory is in the analysis of the linearisation about a balanced metric. Fix an orthornormal basis $s_{\alpha}$ of $H^{0}\left(L^{k}\right)$ for the balanced metric. Suppose $G_{\alpha \beta}=\delta_{\alpha \beta}+\epsilon_{\alpha \beta}$ is another metric. Then, to first order in $\epsilon$,

$$
T_{\nu}(G)=R \int_{X} \frac{\left(s_{\alpha}, s_{\beta}\right)}{1-\sum \epsilon_{\alpha \beta}\left(s_{\alpha}, s_{\beta}\right)} d \nu,
$$

so we have

$$
T_{\nu}(G)=\delta_{\alpha \beta}+\tilde{\epsilon}_{\alpha \beta}+O\left(\epsilon^{2}\right),
$$

where

$$
\tilde{\epsilon}_{\alpha \beta}=R \int_{X} \sum_{\gamma \delta}\left(s_{\alpha}, s_{\beta}\right)\left(s_{\gamma}, s_{\delta}\right) \epsilon_{\gamma \delta} d \nu,
$$

so $\tilde{\epsilon}=Q(\epsilon)$. In other words, the linearisation of the map $T$ at the balanced metric is given by $Q$. In particular, the largest eigenvalue of $Q$ on the trace-free matrices is the quantity $\sigma$ which determines the asymptotic rate of convergence of a sequence $T^{r}\left(G_{0}\right)$, for almost all initial conditions $G_{0}$. Thus we can estimate this largest eigenvalue, in the examples discussed above, by analysing this convergence. For example, on the K3 surface $S$ we estimate, by analysing the sequences, that the eigenvalue is approximately .22 when $k=6$ and .33 when $k=9$.

4.2. Refined approximations and the heat kernel. Another way in which the operator $Q$ enters our theory is in the algorithm we have used for finding "refined approximations" as discussed in (2.2.1) above. Recall that the linearisation of the map which assigns the volume form $\mu_{\omega}=\omega^{n} / n$ ! to a Kahler metric $\omega$ is given by one half the Riemannian Laplacian, i.e.

$$
\mu_{\omega+i} \bar{\partial} \partial_{\phi}=\mu_{\omega}\left(1+\frac{1}{2} \Delta \phi\right)+O\left(\phi^{2}\right)
$$

Suppose given any metric $G$ on $H^{0}\left(L^{k}\right)$, defining a metric $\omega$ on $X$, and let $s_{\alpha}$ be an orthonormal basis of sections. Consider a small perturbation of $G$ to a metric with matrix $\delta_{\alpha \beta}+\epsilon_{\alpha \beta}$ in this basis. Then to first order in $\epsilon$ the induced Fubini-Study metric changes by $i \bar{\partial} \partial \phi$ where

$$
\phi=\sum \epsilon_{\alpha \beta}\left(s_{\alpha}, s_{\beta}\right) .
$$

So, to first order, the change in the volume form is

$$
\frac{1}{2} \Delta\left(\sum \epsilon_{\alpha \beta}\left(s_{\alpha}, s_{\beta}\right)\right) \text {. }
$$


Now given a fixed volume form $\nu$ on $X$, write $\mu_{\omega}=\eta \nu$, where we suppose $\eta$ is close to 1. As in (2.2.1) define

$$
\eta_{\alpha \beta}=R \int_{X}(\eta-1)\left(s_{\alpha}, s_{\beta}\right) d \nu,
$$

and consider the variation

$$
\epsilon_{\alpha \beta}=-\kappa \eta_{\alpha \beta} .
$$

Then the change in the volume form is, to first order,

$$
-\frac{\kappa}{2} \Delta Q_{K}(\eta)
$$

Thus the algorithm of (2.2.1) will replace an initial error term $\eta-1$ by a new term which is approximately

$$
W(\eta-1)=\eta-1-\frac{\kappa}{2} \Delta Q_{K}(\eta),
$$

so we would like to argue that, for appropriate values of the parameter $\kappa$ and with respect to a suitable norm, the linear map $W$ is a contraction.

To give evidence for this, we argue that the operator $Q_{K}$ should be related, asymptotically as $k \rightarrow \infty$, to the heat kernel on $X$. Consider the model case of a line bundle over $\mathbf{C}^{n}$ with curvature $-i \omega$, where $\omega$ is the standard Kahler form (corresponding to the Euclidean metric). Fix a trivialisation of the line bundle in which the connection form is $\frac{-i}{2} \sum\left(x_{a} d y_{a}-y_{a} d x_{a}\right)$ where $z_{a}=x_{a}+i y_{a}$ are standard co-ordinates on $\mathbf{C}^{n}$. Then, in this trivialisation, the section $\sigma_{0}$ which represents evaluation at 0 is

$$
\sigma_{0}=\frac{1}{(2 \pi)^{n}} e^{-|z|^{2} / 4}
$$

so our kernel is

$$
K(0, z)=\frac{1}{(2 \pi)^{2 n}} e^{-|z|^{2} / 2} .
$$

The Euclidean heat kernel is

$$
H(0, z, t)=\left(\frac{1}{4 \pi t}\right)^{n} e^{-|z|^{2} / 4 t},
$$

so $K(0, z)=(2 \pi)^{-n} H(0, z, 1 / 2)$. Thus it is reasonable to expect that, on a general manifold $X$ the operator $Q_{K}$ will be approximately $e^{-\Delta / 2}$ when $k$ is large; so the manifold has very large volume and the local geometry approaches the Euclidean case. (Notice that the factor of $(2 \pi)^{n}$ is accounted for by the scaling built into the definition of $Q_{K}$, since when $k$ is large, by Riemann-Roch $\operatorname{dim} H^{0}\left(L^{k}\right)$ is approximately $(2 \pi)^{-n}$ times the volume, in the metric defined by the curvature 
form of $L^{k}$.) Results very close to this appear in [5]. After this article was posted in preprint form, Liu and Ma proved in [10] a precise result, spelling out the relation between the operators $Q_{K}$ and $e^{-\Delta / 2}$. Thus, while our present purpose is merely to give a plausible justification for the method rather than a rigorous proof, it seems likely that the results of [10] could be used as a basis for a rigorous analysis.

With this discussion in place, we argue that near to the Calabi-Yau metric, the operator $W$ is approximately

$$
\tilde{W}=1-\kappa \frac{\Delta}{2} e^{-\Delta / 2} .
$$

Now $\tilde{W}$ is easy to analyse in terms of the spectrum of the Laplacian. On an eigenspace belonging to eigenvalue $\mu, \tilde{W}$ acts as $\left(1-\kappa \frac{\mu}{2} e^{-\mu / 2}\right)$. Since the function $x e^{-x}$ has maximum value $e^{-1}$ for positive $x$, the operator $\tilde{W}$ is a contraction provided that $0<\kappa<2 e$. This is consistent with the values of the parameter $\kappa$ found to be effective empirically. These ideas also explain why the "refining algorithm" takes a long time to get very close to the refined approximation, since the contraction factor for large eigenvalues $\mu$ is extremely close to 1 .

As a byproduct of these ideas, we can hope to get information about the spectrum of the Laplacian of the Calabi-Yau metric from our theory. Let $\Delta_{0}$ be the Laplacian of the metric scaled to have total volume $(2 \pi)^{n}$ and write

$$
k^{\prime}=\left(\operatorname{dim} H^{0}\left(L^{k}\right)\right)^{1 / n} .
$$

Then we expect that the spectrum of $Q$ approximates that of $e^{-\Delta_{0} / 2 k^{\prime}}$. Thus if $\lambda$ is the first eigenvalue of $\Delta_{0}$ we expect that the convergence parameter $\sigma_{k}$ associated to our algorithm is approximately $e^{-\lambda / 2 k^{\prime}}$. If, as in this paper, we work with $\Gamma$-invariant metrics then we should take $\lambda$ to be the first eigenvalue on $\Gamma$-invariant functions. Our estimates $\sigma_{6}=.22, \sigma_{9}=.33$ are reasonably consistent with this since

$$
-2 \log (.22)(38)^{1 / 2}=18.7
$$

and

$$
-2 \log (.33)(83)^{1 / 2}=20.2 .
$$

So we expect that $\lambda$ is about 20. (We can also hope to get approximations to the eigenfunctions of the Laplacian from the eigenvectors of Q.) 
4.3. Algebraic approximation to the heat kernel. We have now explained the importance of the finite-dimensional linear operator $Q$ in our theory, and its relation to the Laplace operator on the manifold. Recall that the matrix entries of $Q$, in terms of an orthonormal basis $s_{\alpha}$ of $H^{0}\left(L^{k}\right)$, are

$$
Q_{\alpha \beta, \gamma \delta}=R \int_{X}\left(s_{\beta}, s_{\alpha}\right)\left(s_{\gamma}, s_{\delta}\right) d \nu
$$

On the face of it, this requires us to evaluate the large number $\operatorname{dim} H^{0}\left(L^{k}\right)^{4}$ of integrals over $X$ to find the matrix. However we can write

$$
Q_{\alpha \beta, \gamma \delta}=R \int_{X}\left(s_{\beta} s_{\gamma}, s_{\alpha} s_{\delta}\right) d \nu
$$

where the products are sections of $L^{2 k}$ and (, ) denotes the fibre metric on $L^{2 k}$. Let $\tau_{i}$ be a basis of $H^{0}\left(L^{2 k}\right)$. If we know the integrals

$$
I_{i j}=\int_{X}\left(\tau_{i}, \tau_{j}\right) d \nu
$$

then we can compute the matrix entries in terms of purely algebro-geometric data: the product map

$$
H^{0}\left(L^{k}\right) \otimes H^{0}\left(L^{k}\right) \rightarrow H^{0}\left(L^{2 k}\right)
$$

Explicitly, if

$$
s_{\alpha} s_{\beta}=\sum P_{\alpha \beta i} \tau_{i}
$$

then

$$
Q_{\alpha \beta, \gamma \delta}=\sum_{i j} \bar{P}_{\alpha \delta i} \boldsymbol{\Phi}_{\beta \gamma j} I_{i j}
$$

This means that we only need to evaluate approximately $2^{n}(N+1)^{2}$ integrals to find the matrix. Moreover these integrals are precisely the integrals which define the map $T$ for the line bundle $L^{2 k}$. In geometric terms, for any vector space $V$ we have the Veronese embedding

$$
\mathbf{P}(V) \rightarrow \mathbf{P}\left(s^{2} V\right) .
$$

A hermitian metric on $V$ defines a standard induced metric on $s^{2} V$ and, up to a scale factor, the Veronese embedding is isometric with respect to the Fubini-Study metrics. Thus for $X$ in $\mathbf{P}(V)$ we get the same induced metric by embedding in $\mathbf{P}\left(s^{2}(V)\right)$. Starting with the canonical embedding in $V=H^{0}\left(L^{k}\right)^{*}$ we get the canonical embedding in $\mathbf{P}\left(H^{0}\left(L^{2 k}\right)^{*}\right)$, which is contained as a linear subspace in $\mathbf{P}\left(s^{2} V\right)$. Thus, starting with a metric $G$ on $H^{0}\left(L^{k}\right)$ we take the standard induced 
metric $G^{\prime}$ on $H^{0}\left(L^{2 k}\right)$, regarded as a quotient of the symmetric product. Then the calculation of $T\left(G^{\prime}\right)$ is equivalent to the calculation of the matrix entries $I_{i j}$.

Now suppose it happens that $G$ is the balanced metric for $L^{k}$ and $G^{\prime}$ is also the balanced metric for $L^{2 k}$. This will only be the case in rather special circumstances, but for example it holds when $X=\mathbf{C P}^{1}$ with $\nu$ equal to the standard area form. In this case we have $T\left(G^{\prime}\right)=G^{\prime}$ and we can find the matrix entries purely algebraically, in terms of the product map Equation 8 and the original hermitian metric $G$. For example for the line bundle $O(k)$ over $\mathbf{C P}^{1}$, restricting to the $S^{1}$-invariant metrics, we get the matrix entries $Q_{i j}$ in Equation 7 above. But in any case we can define another endomorphism $\tilde{Q}$ of $H\left(L^{k}\right)$ by this procedure. That is, we take the matrix enties

$$
\tilde{Q}_{\alpha \beta, \gamma \delta}=\sum_{i j} \bar{P}_{\alpha \delta i} P_{\beta \gamma j} \tilde{I}_{i j}
$$

where $\tilde{I}_{i j}$ are the inner products in $H^{0}\left(L^{2 k}\right)$ given by the induced hermitian metric, regarded as a quotient of the symmetric square. It is reasonable to expect that, when $k$ is sufficiently large, the metric $G^{\prime}$ is close to the balanced metric and hence that $\tilde{Q}$ is a good approximation to $Q$. To sum up, starting with a hermitian metric $G$ on $H^{0}\left(L^{k}\right)$ we have a purely algebraic procedure for defining a self-adjoint endomorphism $\tilde{Q}$ on $H\left(L^{k}\right)$, and when $G$ is the balanced metric (or close to the balanced metric) we can expect that $\tilde{Q}$ is an approximation to the heat kernel $e^{-\Delta_{0} / 2 k^{\prime}}$.

To illustrate these ideas consider first the case of $S^{1}$-invariant metrics on $S^{2}$. The $S U(2)$ invariance of the problem implies that the eigenspaces of $Q_{K}$ correspond to spherical harmonics. Let $z \in[-1,1]$ be the standard height co-ordinate on the sphere and $p \in S^{2}$ be the pole where $z=1$. The kernel function $K(p$, associated to $O(k)$ is proportional to $(1+z)^{k}$ and so the eigenvalue $\chi_{m, k}$ of $Q$ associated to the spherical harmonics of degreee $m$ is

$$
\chi_{m, k}=\frac{k+1}{2^{k+1}} \int_{-1}^{1}(1+z)^{k} P_{m}(z) d z
$$

where $P_{m}$ is the Legendre polynomial. It is an exercise in Legendre polynomials to show that

$$
\chi_{m, k}=\frac{\left(k^{\prime}-1\right) \ldots\left(k^{\prime}-m\right)}{\left(k^{\prime}+1\right) \ldots\left(k^{\prime}+m\right)}
$$


where we write $k^{\prime}=k+1=\operatorname{dim} H^{0}(O(k))$, as above. Now set $\lambda_{m, k}=$ $-2 k^{\prime} \log \left(\chi_{m, k}\right)$, so

$$
\lambda_{m, k}=-2 k^{\prime} \sum_{r=1}^{m}\left(\log \left(1-\frac{r}{k^{\prime}}\right)-\log \left(1+\frac{r}{k^{\prime}}\right)\right) .
$$

From the Taylor expansion of the logarithm we see that

$$
\lambda_{m, k}=2 m(m+1)+O\left(k^{-2}\right),
$$

and the limits $2 m(m+1)$ are the eigenvalues of the Laplacian on the sphere of area $2 \pi$. For example, we have

$$
\lambda_{1,4}=4+.055, \lambda_{2,10}=12+.15, \lambda_{4,30}=40+.14 .
$$

This also ties in with observed value of $\sigma$ in Section $(2.2)$, since $\chi_{2,6}=5 / 12=$ $.41666 \ldots$

Finally we consider the balanced metric on the $K 3$ surface $S$, with $k=6$. We restrict to the $\Gamma$-invariant part of $H\left(L^{k}\right)$. We work using an orthonormal basis of $H^{0}\left(L^{k}\right)$ given by rescaling the standard monomials, apart from the triple $1, x^{6}, y^{6}$. Here we choose scalars $A, B$ such that $A+B x^{6}+B y^{6}$ and the two similar terms given by permutations are orthonormal. Then we find that the endomorphism $\tilde{Q}$ on the 11-dimensional $\Gamma$-invariant part of $H\left(L^{k}\right)$ has matrix

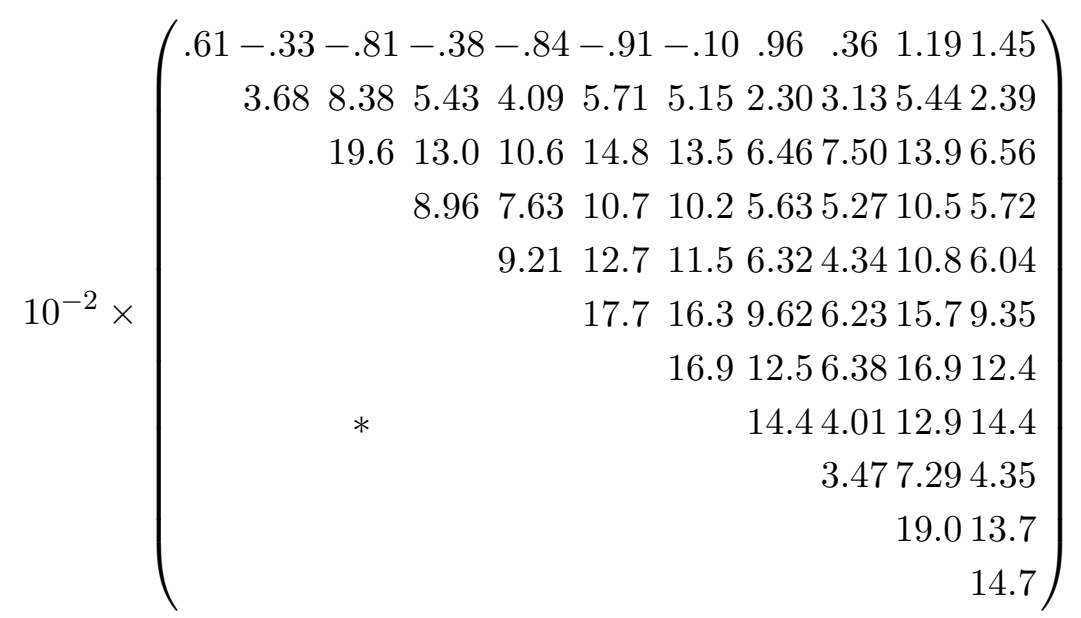

The matrix is symmetric so we omit the entries below the diagonal. Here the first basis element corresponds to the off-diagonal term, the next seven to the entries in the big triangle and the last three to the small triangle. 
We find the first six eigenvalues (ordered by absolute value) of this matrix numerically. They are

$$
1.002, .1956, .05857, .02395,-.002669, .002388 \text {. }
$$

The first eigenvalue, 1.002, is a substitute for the exact eigenvalue 1 of the matrix $Q$, and the close agreement is encouraging. The fact that the fifth eigenvalue is negative, whereas $Q$ is a positive operator, shows that we cannot take the approximation this far down the spectrum. For each positive eigenvalue $\chi$ we compute $\lambda=-2 \sqrt{38} \log \chi$ and for the second, third and fourth eigenvalues we obtain the $\lambda$-values

$$
20.12,34.98,46.00
$$

respectively. The eigenvalue $\chi=.1956$ is in reasonable agreement with our previous numerical estimate .22 for the first eigenvalue of $Q$ and the corresponding estimate 20.12 for the lowest eigenvalue of the Laplacian is very close to our previous estimate 20.2 from the observed value of $\sigma$ when case $k=9$. It is perhaps reasonable to predict, based on this discussion, that the next eigenvalue of the Laplacian $\Delta_{0}$ (on invariant functions) is about 35 . It would be interesting to test this by repeating the work for $k=9$, but the author has not yet had time to do so.

\section{Appendix}

Here we discuss the fact stated in the intoduction, that given any Kahler metric $\omega$ in the class $c_{1}(L)$ there is a sequence of "algebraic" metrics $\omega_{k}$ arising from Hermitian forms on $H^{0}\left(L^{k}\right)$, with $\omega_{k}-\omega=o\left(k^{\nu}\right)$ for all $\nu$.

The proof uses the Tian-Zelditch-Lu expansion and is similar to the argument in [6]. We start with Tian's approximation which, in the notation of Section 2, is to take $F S \circ \operatorname{Hilb}(\omega)$. (Here we are regarding $k$ as a parameter which is supressed in the notation.) Then

$$
F S \circ \operatorname{Hilb}(\omega)=\omega+k^{-1} i \bar{\partial} \partial \log \left(\rho_{\omega}\right)
$$

where $\rho_{\omega}$ is the density of states function $\sum\left|s_{\alpha}\right|^{2}$ for an orthonormal basis $s_{\alpha}$. We know that $\rho_{\omega}$ has an asymptotic expansion

$$
\rho_{\omega}=1+k^{-1} a_{1}(\omega)+k^{-2} a_{2}(\omega)+\ldots,
$$


for certain local invariants $a_{i}$ of the Kahler metric $\omega$. Thus $\tilde{\omega}-\omega=O\left(k^{-2}\right)$ and the order $k^{-2}$ term is $i \bar{\partial} \partial a_{1}(\omega)$. Now let $\omega^{*}=\omega-k^{-2} i \bar{\partial} \partial a_{1}(\omega)$ and consider the metric $F S \circ H i l b\left(\omega^{*}\right)$. Applying the expansion, with smooth dependence on parameters, we see that

$$
F S \circ \operatorname{Hilb}\left(\omega^{*}\right)=\omega+O\left(k^{-3}\right) .
$$

We can repeat this process to kill of successively as many terms as we please in the asymptotic expansion. The correction terms will become progressively more complicated, involving contributions from the derivatives of $a_{i}(\omega)$ with respect to $\omega$, just as in [6]. In this way we obtain, for any given $\nu$, a seqence of approximations $\omega_{k}$ with $\omega_{k}=\omega+o\left(k^{\nu}\right)$. A standard "diagonal' argument gives a single sequence with difference $o\left(k^{\nu}\right)$ for any $\nu$.

There is an elementary argument to prove a somewhat weaker result. The construction of a Fubini-Study metric $\omega_{H}$ from a Hermitian form $H$ can be extended to allow indefinite forms $H$, so long as $H$ is positive on the vectors in $H^{0}\left(L^{k}\right)^{*}$ corresponding to points of $X$. It is easy to prove that any Kahler metric can be rapidly approximated by algebraic metrics in this larger class. It is convenient to assume that $L$ is a very ample line bundle over $X$, so the sections of $L$ give an embedding of $X$ in $\mathbf{C P}^{N}$. (The argument can be extended to avoid this assumption.) For $k \geq 1$ consider the standard Veronese embedding

$$
\mathbf{C P}^{N} \rightarrow \mathbf{C P}^{N_{k}} \text {. }
$$

Let $Z_{A}$ denote standard homogeneous co-ordinates on $\mathbf{C P}^{N_{k}}$ and let $V_{k} \subset C^{\infty}\left(\mathbf{C P}^{N}\right)$ be the vector space of complex-valued functions on $\mathbf{C P}^{N}$ given by linear combinations of

$$
\frac{Z_{A} \overline{Z_{B}}}{|z|^{2 k}}
$$

Of course the $Z_{A}$ are just the monomials of degree $k$ in the homogeneous coordinates $z_{\alpha}$ on $\mathbf{C P}^{N}$.

Lemma 1. The space $V_{k}$ is the direct sum of the first $k$ eigenspaces of the Laplace operator $\Delta_{\mathbf{C P}^{N}}$ for the standard Fubini-Study metric on $\mathbf{C P}^{N}$.

To prove this we take as known the analogous and well-known fact for the Laplacians on spheres. The sum of the first $k$ eigenspaces for the Laplacian on $S^{m-1} \subset \mathbf{R}^{m}$ is exactly the space of functions given by restrictions of polynomials of degree $k$ on $\mathbf{R}^{m}$. Now consider the Hopf fibration $S^{2 N+1} \rightarrow \mathbf{C} \mathbf{P}^{N}$. This is a 
Riemannian submersion so eigenfunctions of the Laplacian on $\mathbf{C P}^{N}$ lift to $S^{1}$ invariant eigenfunctions on the sphere. So the sum of the first $k$ eigenspaces on $\mathbf{C P}^{N}$ can be identified with the polyomials in $z_{\alpha}, \overline{z_{\alpha}}$ which are $S^{1}$-invariant. But it is clear that these are just polynomials in the products $z_{\alpha} \overline{z_{\beta}}$. Separating out the holomorphic and antiholomorphic terms, we see that these are exactly the linear combinations of the products $Z_{A} \overline{Z_{B}}$, as required.

Now it is a standard fact that if $f$ is a smooth function on a compact Riemannian manifold and $f_{k}$ is the $L^{2}$ projection of $f$ to the sum of the first $k$ eigenspaces of the Laplacian then $f_{k}-f=o\left(k^{\nu}\right)$ for any $\nu$. Let $\omega_{0}$ be the metric on $X$ given by the restriction of the standard Fubini-Study metric on $\mathbf{C P}^{N}$ so

$$
\omega=\omega_{0}+i \bar{\partial} \partial \phi
$$

for some smooth function $\phi$ on $X$. Extend $\phi$ arbitrarily to a smooth function on $\mathbf{C P}^{N}$ and take $f=e^{\phi}$. Then $f$ is a positive real valued function on $\mathbf{C P}^{N}$ and there is no loss in generality in supposing that the projections $f_{k}$ are also positive on $\mathbf{C P}^{N}$. By the Lemma the function $f_{k}$ is a sum

$$
\frac{\sum h_{A B} Z_{A} \overline{Z_{B}}}{|z|^{2 k}} \text {. }
$$

and the Fubini-Study metric $\omega_{k}$ associated to the form with matrix $\delta_{A B}+h_{A B}$ is $\omega+i \bar{\partial} \partial\left(\log f_{k}-\log f\right)$. So $\omega_{k}-\omega$ is $o\left(k^{\nu}\right)$ for all $\nu$.

\section{REFERENCES}

[1] D. Acheson From calculus to chaos Oxford U.P. 1997

[2] C. Arezzo, A. Ghigi and A. Loi Stable bundles and the first eigenvalue of the Laplacian Jour. Geom. Analysis 17 (2007) 375-86

[3] J-P. Bourguignon, P.Li and S-T. Yau Upper bounds for the first eigenvalue of algebraic submanifolds Comment. Math. Helvetici 69 (1994) 199-207

[4] E. Calabi Extremal Kahler metrics In:Seminar on Differential Geometry S-T. Yau ed., Princeton U.P. 1983

[5] X Dai, K. Liu and X. Ma On the asymptotic expansion of the Bergman kernel C.R. Acad. Sci. Paris Ser.I 339 (2004) 193-98

[6] S. Donaldson Scalar curvature and projective embeddings, I Jour. Differential Geometry 58 (2001) 479-522

[7] S. Donaldson Scalar curvature and projective embeddings, II Quarterly Jour. Math. 56 (2005) 345-56

[8] M. Headrick and T. Wiseman Numerical Ricci-flat metrics on K3 Classical Quantum Gravity 22 (2005) 4931-69 
[9] H. Luo Geometric criterion for Mumford-Gieseker stability of polarised manifold Jor. Differential Geometry 49 (1998) 577-99

[10] K. Liu and X. Ma A remark on "Some numerical results in complex differential geometry" Math. Res. Letters 14 (2007) 165-71

[11] T. Mabuchi An energy-theoretic approach to the Hitchin-Kobayashi correspondence for manifolds Invent. Math. 159 (2004) 225-243

[12] J. Millson and B. Zombro A Kahler structure on the moduli space of isometric maps of a circle into Euclidean space Invent. Math. 123 (1996) 35-59

[13] D. Mumford Geometric Invariant Theory Springer 1982

[14] P. Newstead Introduction to moduli problems and orbit spaces Tata Institute Lectures 51, Springer 1978

[15] Y. Sano Numerical algorithm for finding balanced metrics Osaka J. Math. 43 (2006) 679-88

[16] G. Tian On a set of polarised Kahler metrics on algebraic manifolds J. Differential Geometry 32 (1990) 99-130

[17] S. Zhang Heights and reductions of semistable varieties Compositio Math. 104 (1996) 77-105

S. K. Donaldson

Department of Mathematics

Imperial College, 180 Queen's Gate

London SW7 2AZ

England

E-mail: s.donaldson@imperial.ac.uk 